\title{
Vector Curvaton with varying Kinetic Function
}

\author{
Konstantinos Dimopoulos, 1 , 田 Mindaugas Karčiauskas,, , 田 and Jacques M. Wagstaff ${ }^{1}$, 团 \\ ${ }^{1}$ Physics Department, Lancaster University, Lancaster LA1 4YB, U.K.
}

(Dated: October 25, 2018)

\begin{abstract}
A new model realisation of the vector curvaton paradigm is presented and analysed. The model consists of a single massive Abelian vector field, with a Maxwell type kinetic term. By assuming that the kinetic function and the mass of the vector field are appropriately varying during inflation, it is shown that a scale invariant spectrum of superhorizon perturbations can be generated. These perturbations can contribute to the curvature perturbation of the Universe. If the vector field remains light at the end of inflation it is found that it can generate substantial statistical anisotropy in the spectrum and bispectrum of the curvature perturbation. In this case the non-Gaussianity in the curvature perturbation is predominantly anisotropic, which will be a testable prediction in the near future. If, on the other hand, the vector field is heavy at the end of inflation then it is demonstrated that particle production is approximately isotropic and the vector field alone can give rise to the curvature perturbation, without directly involving any fundamental scalar field. The parameter space for both possibilities is shown to be substantial. Finally, toy-models are presented which show that the desired variation of the mass and kinetic function of the vector field can be realistically obtained, without unnatural tunings, in the context of supergravity or superstrings.
\end{abstract}

PACS numbers: $98.80 . \mathrm{Cq}$

\section{INTRODUCTION}

Cosmic inflation is the most compelling solution to the horizon and flatness problems of the standard, hot big bang cosmology. As an additional bonus, inflation can also generate the primordial curvature perturbation $\zeta$ of the Universe, which is necessary for the formation of the observed structures such as galaxies and galactic clusters. Fortunately, a snapshot of the primordial curvature perturbation is reflected onto the Cosmic Microwave Background $(\mathrm{CMB})$ radiation, whose temperature perturbations can provide detailed information about $\zeta$, such as the amplitude and scale dependence of its spectrum. The importance of this information lies in its discriminatory power as it offers, so far, the only tool which allows the testing of individual inflation models. Detailed cosmological observations, not only of the CMB but also of the distribution of structures in the Universe (e.g. galaxy surveys), are in agreement with the generic predictions of inflation for $\zeta$. Indeed, the observational evidence supports the existence of a nearly scale-invariant spectrum of predominantly adiabatic and Gaussian superhorizon perturbations. Inflation typically produces such perturbations through the amplification of the quantum fluctuations of suitable fields during a period of accelerated expansion of space. The process is called particle production and it is a manifestation of the Hawking radiation process in de Sitter space. Now that observations of the products of this process are becoming ever more precise, model-builders are forced to construct inflationary models which are better and more closely connected with the theory, more detailed and rigorous, and more testable and falsifiable by comparison with the data. The question to be asked then is, which are those "suitable fields", whose quantum fluctuations are the ultimate sources of the curvature perturbation and all the observed structures in the Universe?

A very useful tool in theoretical physics and, by extension, in cosmology has been the fundamental scalar quantum field. Such scalar fields are ubiquitous in theories beyond the standard model such as supersymmetry (the scalar partners of the observed fermion fields) or string theory (string moduli fields). In cosmology, a scalar field is usually assumed to drive the dynamics of inflation (inflaton field) or even give rise to the accelerated expansion at present (quintessence field). It is not surprising, therefore, that the particle production process, responsible for the generation of $\zeta$, has until recently been considered only in the context of scalar fields. So useful a tool is a scalar field that one tends to forget that, as yet, it is no more than a theoretical construction, whose physical existence is only conjectured, since no fundamental scalar quantum field has ever been observed. One could wonder therefore whether other kinds of fields contributed to the generation of the curvature perturbation in the Universe. What if scalar fields did not

\footnotetext{
*Electronic address: k.dimopoulos1@lancaster.ac.uk

${ }^{\dagger}$ Electronic address: m.karciauskas@lancaster.ac.uk

‡Electronic address: wagstafj@exchange.lancs.ac.uk
} 
exist? Could the galaxies form without them?

Recently, the contribution of vector boson fields to the curvature perturbation is attracting growing attention. In the pioneering work in Ref. [1] a mechanism was introduced, which could potentially allow a single vector field to generate the observed curvature perturbation. The mechanism was the first such suggestion in the literature and was based on the curvaton idea, which was developed for scalar fields in Ref. [2] (see also Ref. [3]) in order to alleviate the fine-tunning problems of inflation model-building [4]. The vector curvaton mechanism assumes that the vector field has a negligible contribution to the energy budget of the Universe during inflation, when on the one hand it becomes homogenised, but on the other hand can also obtain a scale-invariant superhorizon spectrum of perturbations due to some appropriate breaking of its conformality. After inflation the density parameter of the vector field grows, especially after the mass of the vector field becomes important (larger than the Hubble scale). When this happens, the zero-mode undergoes coherent oscillations during which the vector field acts as pressureless, isotropic matter 1]. Hence, it can dominate (or nearly dominate) the Universe without generating excessive anisotropic expansion, avoiding thus the primary obstacle for using vector fields to generate $\zeta$. When the field dominates the Universe it imposes its curvature perturbation spectrum according to the curvaton scenario [2].

The vector curvaton mechanism is a paradigm in search of a model, much like inflation itself. The main ingredient necessary is a mechanism for the generation of a flat spectrum of vector field perturbations during inflation. This is a problem which was originally addressed by efforts to generate a coherent primordial magnetic field during inflation, in order to source the magnetic fields of the galaxies (for a review see Ref. [5]). As realised in Ref. [6] , the particle production process can operate with vector fields only when their conformal invariance is appropriately broken. A number of proposals have been put forward for this to occur during inflation [7 9$]$. For example, as discussed in Ref. [1], a flat spectrum of vector field perturbations is generated if the effective mass-squared of the vector field during inflation is $m_{\text {eff }}^{2}=-2 H_{*}^{2}$, where $H_{*}$ is the inflationary Hubble scale. In Ref. [6] it has been shown that this could be achieved if the vector field is non-minimally coupled to gravity with a coupling of the form $\frac{1}{6} R A^{2}$, where $R$ is the Ricci scalar. This idea was employed in the vector curvaton mechanism in Ref. [10]. Another possibility was to consider a varying kinetic function as discussed in Ref. [9]. The first attempt to implement this idea to the vector curvaton mechanism is in Ref. [11].

In the above early works on the vector curvaton, attention was paid mainly on the transverse components of the vector field perturbations. The reason was that the vector curvaton mechanism employs the fractional perturbation of the density of the vector field, which is a scalar quantity, i.e. isotropic. Indeed, the mechanism does not cause any anisotropic expansion because, when the zero-mode of the field undergoes rapid coherent oscillations, so do the field's perturbations. Thus, the transverse components were deemed enough to generate $\zeta$ and the longitudinal component was ignored. This turned out to miss a key effect introduced to the curvature perturbation from vector fields, namely statistical anisotropy.

Statistical anisotropy in the curvature perturbation is a new observable and it amounts to direction dependent patterns in the CMB temperature perturbations as well as in the large scale structure (e.g. rows of galaxies) if it is intense enough. The observational limit on such a signal is rather weak as statistical anisotropy in the spectrum of $\zeta$ is allowed up to as much as $30 \%$ [12]. This implies that it is likely to be observed by the forthcoming observations of the Planck satellite mission which will detect statistical anisotropy in the spectrum if it is larger than $2 \%$ [13], while there is no reason to think that this will be the ultimate limit. Another possible indication of statistical anisotropy in the curvature perturbation is the alignment of the quadrupole and octupole moments of the CMB (the so-called "Axis of Evil" [14]) and of galaxy spins [15, 16]. The alignment of the low CMB multipoles was shown to persist beyond foreground removal [17] despite being statistically extremely unlikely with isotropic perturbations (see also [18]). Moreover, in Ref. [16] it is claimed that the preferred direction of the alignment of galactic spins appears to coincide with the "Axis of Evil", as one would expect if they were both due to statistical anisotropy in $\zeta$. It has to be noted that statistical anisotropy cannot be introduced by scalar fields alone, so if its observation is confirmed, this would be a powerful indication that vector fields at least affect $\zeta$.

The first study of the generation of statistical anisotropy by a vector field is in Ref. [19], where a vector field is assumed to modulate the decay of the inflaton. In a separate development [20] it was pointed out that even a homogeneous vector field will generate statistical anisotropy in $\zeta$, because it will break the statistical isotropy of the scalar field perturbation generated from the vacuum fluctuation. This is further explored in Ref. 21]. A comprehensive model-independent study of statistical anisotropy can be found in Ref. 22. The study employs the $\delta N$ formalism [23], extending it to include the effects of vector fields. In Ref. [22] it is shown that a vector field can alone generate $\zeta$ through the vector curvaton mechanism only if the particle production process is approximately isotropic. Otherwise, the contribution of the vector curvaton field has to be subdominant and its significance amounts to the generation of statistical anisotropy only. As an application, the $\frac{1}{6} R A^{2}$ vector curvaton model was revisited and shown to result in statistical anisotropy of order unity, which would be excessive if the vector field were to provide the dominant contribution to $\zeta$. In such a case, the only way that excessive statistical anisotropy can be avoided without introducing another dominant (and statistically isotropic) source of curvature perturbations (such as scalar 
fields) is by considering either a triad of identical vector fields each perpendicular to the other two 24] or hundreds of identical vector fields, randomly oriented in space. In such a case anisotropy in the spectrum or in the expansion can be avoided or suppressed, which means that vector fields could also act as inflatons, without generating anisotropic expansion.

Using vector fields as inflatons was first considered in Ref. [25]. More recently, vector field inflation has been studied in Ref. [26] with the $\frac{1}{6} R A^{2}$ model, involving hundreds of randomly oriented vector fields. For more studies of similar vector inflation models see Refs. [27, 28]|. ${ }^{1}$ In the same spirit, vector fields have been considered as candidates for dark energy [28, 30]. Furthermore, Refs. [31] and 32] investigate inflation or dark energy respectively, using Yang-Mills fields. Finally, inflation with $P$-forms is explored in Ref. [33].

Also obtained in Ref. 22 is the contribution of vector fields in the bispectrum of the curvature perturbation (for the trispectrum see Ref. [34]). Using these results, in Ref. [35] the non-Gaussianity in the spectrum is studied in a model-independent way, though the results are applied to the models of Refs. [10] and [19]. A recent study in Ref. [36], extends this treatment in the case of non-Abelian vector fields, applying the results in the $\frac{1}{6} R A^{2}$ model. In all cases, it is shown that statistical anisotropy in the spectrum and bispectrum are correlated. This is a smoking gun for the contribution of a vector field to the curvature perturbation. It is likely that the Planck mission will observe non-zero non-Gaussianity in the CMB temperature perturbations. If this is the case then simple single-scalar-field models of inflation will be ruled out. If an angular modulation of the non-linearity parameter $f_{\mathrm{NL}}$, which quantifies non-Gaussianity, is also observed, then this is a strong indication of the contribution of vector fields to $\zeta$.

An important aspect of the mechanisms which break the conformality of vector fields and result in particle production of their perturbations during inflation is the appearance of instabilities. For example, in Ref. [37] the $\frac{1}{6} R A^{2}$ model was criticised for giving rise to ghosts and other instabilities, e.g. when the modes of the perturbations exit the horizon, although in Ref. 22] the latter was demonstrated not to be a problem (also see Ref. 22] for a discussion on ghosts). For a comprehensive analysis on ghosts and tachyon instabilities see also Ref. [38].

In this paper we introduce a new model of vector curvaton, which does not suffer from such instabilities (see also Ref. [39]). The model consists of a massive Abelian vector field with a Maxwell type kinetic term, whose kinetic function is modulated during inflation similarly to the models in Ref. [9]. Our model is a follow up of Ref. [11], which did not consider statistical anisotropy. We attempt a detailed analytical investigation and confirm our findings numerically. As we demonstrate, our vector curvaton model gives rise to a rich phenomenology. In particular, when the mass of the vector field is smaller than the inflationary Hubble scale the vector curvaton can give rise to statistical anisotropy in both the spectrum and bispectrum, with the angular modulation dominating $f_{\mathrm{NL}}$, rendering thus the model, in this case, falsifiable by observations in the near future. When the mass of the vector field is larger than the inflationary Hubble scale though, particle production is isotropic and the vector field can alone generate the observed curvature perturbation. The parameter space for both regimes is shown to be substantial.

The structure of our paper is as follows. In Sec. II we present our model and obtain the equations of motion of the perturbations of the vector field components in momentum space. In Sec. III we study particle production in order to obtain the desired scaling of the mass and the kinetic function of the vector field, which results in the production of a scale invariant superhorizon spectrum of perturbations. In Secs. IV and V we calculate in detail the power spectra of the perturbations in the two possible alternatives for the scaling of the kinetic function. To this end we obtain the evolution of the mode functions of the perturbations, through analytic approximations of high precision. In Sec. VI we calculate the statistical anisotropy in the spectrum and bispectrum of the produced perturbations in all cases. In Sec. VII we study the evolution of the zero mode of the vector boson condensate, during and after inflation. The evolution of the zero mode is necessary in order to determine the cosmology after inflation as well as calculate the curvature perturbation. In Sec. VIII we apply the vector curvaton mechanism in order to obtain constraints on the model parameters such as the mass of the vector field and the inflationary Hubble scale. In Sec. IX we present two toy-models which demonstrate how the desired scaling of the mass and the kinetic function of the vector field can be obtained in a natural way in the context of supergravity and superstrings. In Sec. X we summarise and discuss our findings. Finally, in Sec. XI we present our conclusions. Throughout the paper we use natural units such that $c=\hbar=k_{B}=1$ and Newton's gravitational constant is $8 \pi G=m_{P}^{-2}$, where $m_{P}=2.44 \times 10^{18} \mathrm{GeV}$ is the reduced Planck mass.

\footnotetext{
1 Another way to avoid anisotropy is to consider a time-like vector field [29].
} 


\section{EQUATIONS OF MOTION}

Consider the Lagrangian density for a massive, Abelian vector field

$$
\mathcal{L}=-\frac{1}{4} f F_{\mu \nu} F^{\mu \nu}+\frac{1}{2} m^{2} A_{\mu} A^{\mu}
$$

where $f$ is the kinetic function, $m$ is the mass and the field strength tensor is $F_{\mu \nu}=\partial_{\mu} A_{\nu}-\partial_{\nu} A_{\mu}$. The above can be the Lagrangian density of a massive Abelian gauge field, in which case $f$ is the gauge kinetic function. However, we need not restrict ourselves to gauge fields only. If no gauge symmetry is considered the argument in support of the above Maxwell type kinetic term is that it is one of the few (three) choices [38] which avoids introducing instabilities, such as ghosts 37]. Also, we note here that a massive vector field which is not a gauge field is renormalizable only if it is Abelian [40].

We focus, at first, on a period of cosmic inflation, during which we assume that the contribution of the vector field to the energy budget of the Universe is minute and can be ignored. Thus, we take the inflationary expansion to be isotropic. We also assume that inflation is of quasi-de Sitter type, i.e. the Hubble parameter is $H \approx$ constant. We will consider that, during inflation, $f=f(t)$ and $m=m(t)$ can be functions of cosmic time $t$.

Inflation is expected to homogenise the vector field. Following Ref. [11], we find that the temporal component of the homogeneous vector field has to be zero, while the spatial components satisfy the equation of motion:

$$
\ddot{\boldsymbol{A}}+\left(H+\frac{\dot{f}}{f}\right) \dot{\boldsymbol{A}}+\frac{m^{2}}{f} \boldsymbol{A}=0,
$$

where the dot denotes derivative with respect to $t$. From the above it is evident that the effective mass of the vector field is

$$
M \equiv \frac{m}{\sqrt{f}}
$$

where we assumed that $m, f>0$.

In order to study particle production we need to perturb the vector field around the homogeneous zero mode $A_{\mu}(t)$ as:

$$
A_{\mu}(t, \boldsymbol{x})=A_{\mu}(t)+\delta A_{\mu}(t, \boldsymbol{x}) .
$$

Now, let us switch to momentum space by Fourier expanding the perturbations:

$$
\delta A_{\mu}(t, \boldsymbol{x})=\int \frac{d^{3} k}{(2 \pi)^{3 / 2}} \delta \mathcal{A}_{\mu}(t, \boldsymbol{k}) \exp (i \boldsymbol{k} \cdot \boldsymbol{x}) .
$$

Then, according to Ref. [11] the equations of motion for the spatial components of the vector field perturbations in momentum space are:

$$
\left[\partial_{t}^{2}+\left(H+\frac{\dot{f}}{f}\right) \partial_{t}+\frac{m^{2}}{f}+\left(\frac{k}{a}\right)^{2}\right] \delta \mathcal{A}^{\perp}=0
$$

and

$$
\left\{\partial_{t}^{2}+\left[H+\frac{\dot{f}}{f}+\left(2 H+2 \frac{\dot{m}}{m}-\frac{\dot{f}}{f}\right) \frac{\left(\frac{k}{a}\right)^{2}}{\left(\frac{k}{a}\right)^{2}+\frac{m^{2}}{f}}\right] \partial_{t}+\frac{m^{2}}{f}+\left(\frac{k}{a}\right)^{2}\right\} \delta \mathcal{A}^{\|}=0
$$

where $a=a(t)$ is the scale factor of the Universe, $k \equiv|\boldsymbol{k}|$ and the longitudinal and transverse components are defined as:

$$
\delta \mathcal{A}^{\|} \equiv \frac{\boldsymbol{k}(\boldsymbol{k} \cdot \delta \mathcal{A})}{k^{2}} \quad \text { and } \quad \delta \mathcal{A}^{\perp} \equiv \delta \mathcal{A}-\delta \mathcal{A}^{\|} .
$$

To continue we need to employ the canonically normalised vector field $B_{\mu}$ and the physical (in contrast to comoving) vector field $W_{\mu}$, whose spatial components are:

$$
\boldsymbol{B} \equiv \sqrt{f} \boldsymbol{A} \text { and } \boldsymbol{W} \equiv \boldsymbol{B} / a=\sqrt{f} \boldsymbol{A} / a .
$$


Expressing Eqs. (6) and (7) in terms of the physical vector field we obtain the following equations for the spatial components of the physical vector field perturbations:

$$
\left\{\partial_{t}^{2}+3 H \partial_{t}+\frac{1}{2}\left[\frac{1}{2}\left(\frac{\dot{f}}{f}\right)^{2}-\frac{\ddot{f}}{f}-\frac{\dot{f}}{f} H+4 H^{2}\right]+\frac{m^{2}}{f}+\left(\frac{k}{a}\right)^{2}\right\} \delta \mathcal{W}^{\perp}=0
$$

and

$$
\begin{array}{r}
\left\{\partial_{t}^{2}+\left[3 H+\left(2 H+2 \frac{\dot{m}}{m}-\frac{\dot{f}}{f}\right) \frac{\left(\frac{k}{a}\right)^{2}}{\left(\frac{k}{a}\right)^{2}+\frac{m^{2}}{f}}\right] \partial_{t}+\frac{1}{2}\left[\frac{1}{2}\left(\frac{\dot{f}}{f}\right)^{2}-\frac{\ddot{f}}{f}-\frac{\dot{f}}{f} H+4 H^{2}\right]+\right. \\
\left.\left(H-\frac{1}{2} \frac{\dot{f}}{f}\right)\left(2 H+2 \frac{\dot{m}}{m}-\frac{\dot{f}}{f}\right) \frac{\left(\frac{k}{a}\right)^{2}}{\left(\frac{k}{a}\right)^{2}+\frac{m^{2}}{f}}+\frac{m^{2}}{f}+\left(\frac{k}{a}\right)^{2}\right\} \delta \mathcal{W}^{\|}=0,
\end{array}
$$

where

$$
\delta \boldsymbol{W}(t, \boldsymbol{x})=\int \frac{d^{3} k}{(2 \pi)^{3 / 2}} \delta \mathcal{W}(t, \boldsymbol{k}) \exp (i \boldsymbol{k} \cdot \boldsymbol{x})
$$

\section{PARTICLE PRODUCTION}

In this section we attempt a preliminary study of the particle production process during inflation in order to ascertain what kind of time dependence do $f(t)$ and $m(t)$ need to have in order to result in scale-invariant power spectra for all the components of the superhorizon vector field perturbations.

To study particle production of the vector field during inflation, we first need to promote $\delta \boldsymbol{W}$ to a quantum operator. To do that we expand in creation and annihilation operators as

$$
\delta \hat{\boldsymbol{W}}=\int \frac{d^{3} k}{(2 \pi)^{3}} \sum_{\lambda}\left[\boldsymbol{e}_{\lambda}(\hat{\boldsymbol{k}}) \hat{a}_{\lambda}(\boldsymbol{k}) w_{\lambda}(t, k) e^{i \boldsymbol{k} \cdot \boldsymbol{x}}+\boldsymbol{e}_{\lambda}^{*}(\hat{\boldsymbol{k}}) \hat{a}_{\lambda}^{\dagger}(\boldsymbol{k}) w_{\lambda}^{*}(t, k) e^{-i \boldsymbol{k} \cdot \boldsymbol{x}}\right],
$$

where $\hat{\boldsymbol{k}} \equiv \boldsymbol{k} / k$, with $k \equiv|\boldsymbol{k}|$ and $\lambda=L, R, \|$ with $L, R$ denoting the Left and Right transverse polarisations respectively such that

$$
\left(\delta \mathcal{W}^{\perp}\right)^{2}=\left(\delta \mathcal{W}^{L}\right)^{2}+\left(\delta \mathcal{W}^{R}\right)^{2} .
$$

The polarisation vectors $\boldsymbol{e}_{\lambda}$ can be chosen as

$$
e_{L} \equiv \frac{1}{\sqrt{2}}(1, i, 0), \quad e_{R}=\frac{1}{\sqrt{2}}(1,-i, 0), \quad e_{\|}=(0,0,1),
$$

while we have canonical quantisation with

$$
\left[\hat{a}_{\lambda}(\boldsymbol{k}), \hat{a}_{\lambda^{\prime}}^{\dagger}\left(\boldsymbol{k}^{\prime}\right)\right]=(2 \pi)^{3} \delta\left(\boldsymbol{k}-\boldsymbol{k}^{\prime}\right) \delta_{\lambda \lambda^{\prime}} .
$$

Since Eqs. (10) and (11) are linear they will be satisfied also by the corresponding mode functions $w_{\lambda}(t, k)$. To study particle production in this theory we need to solve these equations with the appropriate initial conditions. After obtaining the solutions we will find the appropriate constraints on $f$ and $m$, which can provide us with a scale invariant spectrum of superhorizon perturbations. The strategy is to begin with the transverse equation (10), since it is the simplest. Conditions obtained by requiring a flat spectrum for the transverse components of the vector field perturbations will hopefully simplify the longitudinal equation (11) as well.

\section{A. The transverse components}

Let us assume that the time dependence of the kinetic function can be parametrised as

$$
f \propto a^{\alpha},
$$


where $\alpha$ is a real constant. We will also assume that $f \rightarrow 1$ at (least by) the end of inflation so that, after inflation, the vector field is canonically normalised. Then, according to Eq. (10) the equation of motion for the transverse mode functions is

$$
\ddot{w}_{L, R}+3 H \dot{w}_{L, R}+\left[-\frac{1}{4}(\alpha+4)(\alpha-2) H^{2}+M^{2}+\left(\frac{k}{a}\right)^{2}\right] w_{L, R}=0,
$$

where we used Eq. (3) and considered that the theory is parity invariant so that $\delta \mathcal{W}_{L}=\delta \mathcal{W}_{R}$, i.e. $w_{L}=w_{R} \equiv w_{L, R}$.

In analogy to the equation of motion for the mode function of a scalar field during quasi-de Sitter inflation, it can be readily deduced that a scale invariant spectrum of perturbations is attained if

$$
\alpha=-1 \pm 3
$$

(i.e. either $f \propto a^{2}$ or $f \propto a^{-4}$ ) and

$$
M_{*} \ll H,
$$

where the star denotes the time when the cosmological scales exit the horizon. The latter condition simply requires that the physical vector field $W_{\mu}$ is effectively massless at that time. ${ }^{2}$ The above condition can be obtained as follows.

Let us define

$$
r \equiv \frac{a M}{k} .
$$

If we assume that the field is effectively massless $M \ll k / a$ (i.e. $r \ll 1$ ) until the end of inflation we can straightforwardly calculate the power spectrum of the perturbations. First, we solve Eq. (18) using the Bunch-Davis vacuum boundary condition

$$
\lim _{\frac{k}{a H} \rightarrow+\infty} w_{L, R}=\frac{a^{-1}}{\sqrt{2 k}} e^{i k / a H},
$$

which is valid well within the horizon, where the inflationary expansion is not felt and one can use flat spacetime quantum field theory. The solution is then

$$
w_{L, R}=\frac{a^{-3 / 2}}{2} \sqrt{\frac{\pi}{H}} e^{i \frac{\pi}{2}\left(\nu+\frac{1}{2}\right)} H_{\nu}^{(1)}(k / a H),
$$

where $H_{\nu}^{(1)}$ is the Hankel function of the first kind and $\nu$ is given by

$$
\nu=\frac{1}{2}|\alpha+1| .
$$

At late times (superhorizon scales) the dominant term in the above solution approaches

$$
\lim _{\frac{k}{a H} \rightarrow 0^{+}} w_{L}=-\frac{i a^{-3 / 2}}{2 \Gamma(1-\nu)} \sqrt{\frac{\pi}{H}} e^{i \frac{\pi}{2}\left(\nu+\frac{1}{2}\right)}\left(\frac{k}{2 a H}\right)^{-\nu} .
$$

Hence, the power spectrum of the perturbations is

$$
\mathcal{P}_{L, R} \equiv \frac{k^{3}}{2 \pi^{2}}\left|\lim _{\frac{k}{a H} \rightarrow 0^{+}} w_{L, R}\right|^{2}=\frac{4 \pi}{[\Gamma(1-\nu)]^{2}}\left(\frac{H}{2 \pi}\right)^{2}\left(\frac{k}{2 a H}\right)^{3-2 \nu} .
$$

As evident, scale invariance is attained when $\nu=3 / 2$, which, in view of Eq. (24), results in the values for $\alpha$ shown in Eq. (19). If $\alpha=-1 \pm 3$ then the above gives

$$
\mathcal{P}_{L, R}=\left(\frac{H}{2 \pi}\right)^{2}
$$

\footnotetext{
2 Note that this is not the same as having $A_{\mu}$ being effectively massless. In the latter case the vector field is approximately conformally invariant and does not undergo particle production. However, the conformality of the massless physical vector field $W_{\mu}$ is broken.
} 
i.e. the spectrum is scale invariant with amplitude given by the Hawking temperature for de Sitter space.

As is shown later on, even if the $r \ll 1$ condition is violated before the end of inflation (but after the cosmological scales exit the horizon), the scale dependence of the spectrum at the vicinity of the cosmological scales is not altered (only its amplitude is). The reason for this is that, when $r \gg 1$, the mass term in Eq. (18) dominates the $k$-dependent term. Consequently, the equation loses its sensitivity on scale dependence, which means that the evolution of the perturbations in the $r \gg 1$ regime will not affect their dependence on scale. Hence, a scale-invariant spectrum will remain so. We demonstrate explicitly this in Sec. IV] Thus, Eqs. (19) and (20) are sufficient for the generation of a scale invariant spectrum of perturbations for the transverse component of our vector field.

\section{B. The longitudinal component}

Let us assume now that the time dependence of $m$ can be parametrised as

$$
m \propto a^{\beta},
$$

where $\beta$ is a real constant. Then, in view of our notation and Eq. (11), the equation of motion for the longitudinal mode function is

$$
\ddot{w}_{\|}+\left(3+\frac{2-\alpha+2 \beta}{1+r^{2}}\right) H \dot{w}_{\|}+\left[-\frac{1}{2}(\alpha-2)\left(\alpha+4+\frac{2-\alpha+2 \beta}{1+r^{2}}\right) H^{2}+\left(\frac{k}{a}\right)^{2}\left(1+r^{2}\right)\right] w_{\|}=0 .
$$

Now, let us make use of Eq. (19), which is necessary to obtain a scale-invariant spectrum for the transverse components. If we assume also that $r \ll 1$, the above equation simplifies to

$$
\ddot{w}_{\|}+(5-\alpha+2 \beta) H \dot{w}_{\|}+\left[-\frac{1}{2}(\alpha-2)(2-\alpha+2 \beta) H^{2}+\left(\frac{k}{a}\right)^{2}\right] w_{\|}=0 .
$$

Similarly to the transverse components, we can solve the above equation by using the vacuum boundary condition, which reads

$$
\lim _{\frac{k}{a H} \rightarrow+\infty} w_{\|}=\gamma \frac{a^{-1}}{\sqrt{2 k}} e^{i k / a H} .
$$

Note that, for the longitudinal component of the vector field perturbations, the vacuum condition is multiplied by the Lorentz boost factor $\gamma$, which takes us from the frame with $\boldsymbol{k}=0$ (where there is no distinction between longitudinal and transverse components) to that of momentum $\boldsymbol{k} \neq 0$. The Lorentz boost factor is

$$
\gamma=\frac{E}{M}=\frac{\sqrt{\left(\frac{k}{a}\right)^{2}+M^{2}}}{M}=\sqrt{1+\frac{1}{r^{2}}}
$$

where we considered that the effective mass of the physical vector field is given by Eq. (3).

Now, assuming that $r \ll 1$ at early times, Eq. (30) remains valid within the horizon, where it can be matched to the vacuum expression in Eq. (31) with $\gamma \simeq 1 / r$. By doing so we obtain

$$
w_{\|}=\frac{a^{-3 / 2}}{r} \sqrt{\frac{\pi}{4 H}} \frac{e^{-i \frac{\pi}{2}\left(\hat{\nu}-\frac{3}{2}\right)}}{\sin (\pi \hat{\nu})}\left[J_{\hat{\nu}}\left(\frac{k}{a H}\right)-e^{i \pi \hat{\nu}} J_{-\hat{\nu}}\left(\frac{k}{a H}\right)\right],
$$

where $J_{\hat{\nu}}$ denotes Bessel function of the first kind, and

$$
\hat{\nu}=\frac{1}{2} \sqrt{9+2(\alpha+1)(2-\alpha+2 \beta)+(2-\alpha+2 \beta)^{2}} .
$$

At late times (superhorizon scales) the dominant term in the above solution approaches

$$
\lim _{\frac{k}{a H} \rightarrow 0^{+}} w_{\|}=-\frac{a^{-3 / 2}}{\Gamma(1-\hat{\nu})} \sqrt{\frac{\pi}{H}} \frac{e^{i \frac{\pi}{2}\left(\hat{\nu}+\frac{3}{2}\right)}}{\sin (\pi \hat{\nu})}\left(\frac{H}{M}\right)\left(\frac{k}{2 a H}\right)^{1-\hat{\nu}}
$$


where we used Eq. (21). Hence, the power spectrum of the perturbations is

$$
\mathcal{P}_{\|} \equiv \frac{k^{3}}{2 \pi^{2}}\left|\lim _{\substack{a H \\ a H}} w_{\|}\right|^{2}=\frac{16 \pi}{\sin ^{2}(\pi \hat{\nu})[\Gamma(1-\hat{\nu})]^{2}}\left(\frac{H}{M}\right)^{2}\left(\frac{H}{2 \pi}\right)^{2}\left(\frac{k}{2 a H}\right)^{5-2 \hat{\nu}}
$$

It is clear that scale invariance is attained when $\hat{\nu}=5 / 2$. If this is so then the above becomes

$$
\mathcal{P}_{\|}=9\left(\frac{H}{M}\right)^{2}\left(\frac{H}{2 \pi}\right)^{2}
$$

i.e. the spectrum is scale invariant with amplitude given by the Hawking temperature for de Sitter space, but also determined by the mass fraction $M / H$ of the physical vector field. As with the case of the transverse components, scale invariance is retained even in the regime when $r \gg 1$ (see Sec. IV).

Now, using Eqs. (19) and (34), one finds that scale invariance (i.e. $\hat{\nu}=5 / 2$ ) requires

$$
\beta=-\frac{1}{2}(3 \pm 5)
$$

We can discard one of the above values as follows. ${ }^{3}$ According to Eqs. (17), (21) and (28)

$$
r \propto a^{1+\beta-\alpha / 2} .
$$

This means that the value $\beta=-4$ is unacceptable, because it implies that $r$ is a decreasing function of $a$ (for $\alpha=-1 \pm 3$ ), which means that our assumption $r \ll 1$ cannot hold true in the subhorizon limit. Hence, we conclude that a scale invariant spectrum for the longitudinal component of the vector field perturbations can be attained only if $\beta=1$, i.e.

$$
m \propto a
$$

In view of the above and Eqs. (27) and (37), we see that, if $\alpha=2$, then (when $r \ll 1$ ) we have

$$
\mathcal{P}_{\|}=\text {constant } \gg \mathcal{P}_{L, R} \quad(\text { for } \alpha=2),
$$

where we considered Eq. (20). On the other hand, if $\alpha=-4$, then $\mathcal{P}_{\|} \propto a^{-6}$. Thus, even though the spectrum is scale invariant, its amplitude decreases in time. Scale invariance is not spoiled by the piling up of more and more perturbations, while they exit the horizon, because their amplitude is reduced accordingly in time, as shown in Fig. 1 . The gradual decrease of $\mathcal{P}_{\|}$implies that, even though $\left(\mathcal{P}_{\|} / \mathcal{P}_{L, R}\right)_{*} \gg 1$, this ratio decreases towards the end of inflation and may allow for the longitudinal and transverse spectra to be eventually comparable.

\section{CASE: $f \propto a^{-4} \& m \propto a$}

In this section we assume the values

$$
\alpha=-4 \quad \& \quad \beta=1
$$

i.e. $f \propto a^{-4}$ and $M \propto a^{3}$ [cf. Eq. (3)]. Using these we perform an analytic calculation of the power spectra for all the components of the superhorizon vector field perturbations generated by the particle production process.

\section{A. The transverse components}

For the choice in Eq, (42), the equation of motion for the transverse mode functions in Eq. (18) becomes

$$
\ddot{w}_{L, R}+3 H \dot{w}_{L, R}+\left(\frac{k}{a}\right)^{2}\left(1+r^{2}\right) w_{L, R}=0
$$

\footnotetext{
3 The ' \pm ' sign in Eq. (19) is uncorrelated with the one in Eq. 38], which means that discarding one of the values of $\beta$ does not imply doing so also for one of the values of $\alpha$.
} 


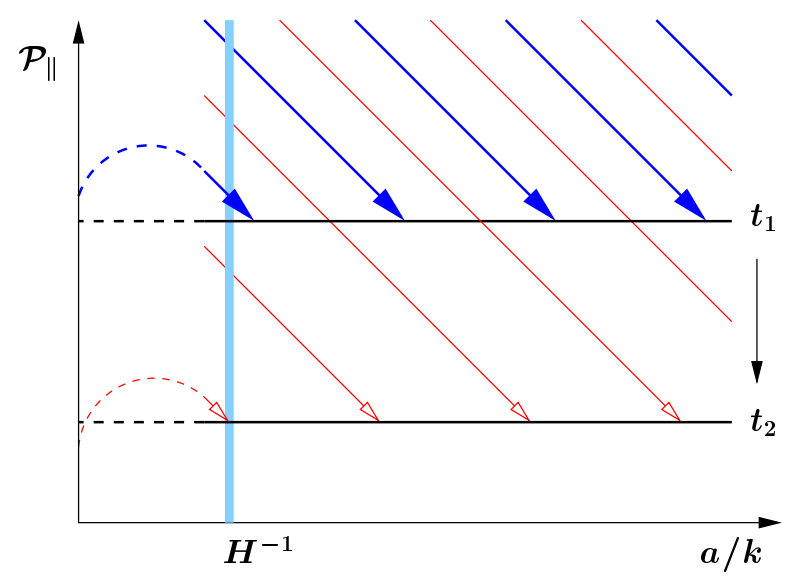

FIG. 1: Log-log plot of the superhorizon power spectrum $\mathcal{P}_{\|} \propto a^{-6}$ (case $\alpha=-4$ ) in terms of the physical lengthscale $\ell \sim a / k$ at a given fixed time $t$. The spectrum is flat and it is shown by the solid horizontal lines, which depict its value at two different times: $t_{1}$ and $t_{2}>t_{1}$ for superhorizon scales $\ell>H^{-1}$. The slanted arrows show the evolution of superhorizon modes of given, fixed $k$. The figure attempts to show that, as time passes and more perturbation modes exit the horizon, their amplitude at horizon crossing reduces in such a way that they end up on top of the flat spectrum at the time of exit.

The generic solution to the above can be obtained when $r \not 1$. Indeed, one finds

$$
\begin{aligned}
& w_{L, R}=a^{-3 / 2}\left[c_{1} J_{3 / 2}\left(\frac{k}{a H}\right)+c_{2} J_{-3 / 2}\left(\frac{k}{a H}\right)\right] \text { for } r \ll 1 \quad \text { and } \\
& w_{L, R}=a^{-3 / 2}\left[\hat{c}_{1} J_{1 / 2}\left(\frac{M}{3 H}\right)+\hat{c}_{2} J_{-1 / 2}\left(\frac{M}{3 H}\right)\right] \text { for } r \gg 1,
\end{aligned}
$$

where $c_{i}$ and $\hat{c}_{i}$ are integration constants. Because of Eq. (20), we find that the switch-over between the two regimes occurs after horizon exit since

$$
\frac{M_{*}}{H} \ll 1 \equiv \frac{k}{a_{*} H} \quad \Rightarrow \quad r_{*} \ll 1
$$

and, in view of Eqs. (39) and (42),

$$
r \propto a^{4}
$$

Also, note that at the switch-over moment, denoted here with subscript $k$ (because it is $k$-dependent), we have

$$
\frac{M_{k}}{H} \equiv \frac{k}{a_{k} H} \ll 1
$$

exactly because the switch-over moment for a given mode occurs when the mode in question is already of superhorizon size.

Using the above we can approximate the solutions in Eqs. (44) and (45) in the vicinity of the switch-over as

$$
w_{L, R}=a^{-3 / 2} \sqrt{\frac{2}{\pi}}\left[\frac{1}{3} c_{1}\left(\frac{k}{a H}\right)^{3 / 2}-c_{2}\left(\frac{a H}{k}\right)^{3 / 2}\right] \text { for } r \lesssim 1 \quad \text { and }
$$




$$
w_{L, R}=a^{-3 / 2} \sqrt{\frac{2}{\pi}}\left[\hat{c}_{1}\left(\frac{M}{3 H}\right)^{1 / 2}+\hat{c}_{2}\left(\frac{3 H}{M}\right)^{1 / 2}\right] \text { for } r \gtrsim 1,
$$

where we used that $k / a_{k} H \ll 1, M_{k} / H \ll 1$ and $\lim _{x \rightarrow 0} J_{\nu}(x)=\frac{x^{\nu}}{2^{\nu} \Gamma(1+\nu)}$.

To approximate the complete solution we can perform a matching at the switch-over of both the mode function $w_{L, R}$ and its time-derivative $\dot{w}_{L, R}$. In effect, this will enable us to connect the $\hat{c}_{i}$ integration constants with the $c_{i}$. To perform the matching it is useful to consider that

$$
\frac{M}{3 H} \propto a^{3} \Rightarrow \frac{a_{k}}{a}=r^{-1 / 4}
$$

After matching we obtain

$$
\hat{c}_{1}=-\frac{\sqrt{3}}{4}\left(\frac{2 a_{k} H}{k}\right)^{2} c_{2} \quad \text { and } \quad \hat{c}_{2}=\frac{4 \sqrt{3}}{9}\left(\frac{k}{2 a_{k} H}\right)^{2} c_{1} .
$$

Now, to evaluate the $c_{i}$ we need to match the solution in Eq. (44) with the vacuum condition in Eq. (22). Thus, we obtain

$$
c_{1}=\frac{1}{2} \sqrt{\frac{\pi}{H}} \quad \text { and } \quad c_{1}=-\frac{i}{2} \sqrt{\frac{\pi}{H}}
$$

Hence, the analytic expression for the transverse mode functions can be written as

$$
\begin{gathered}
w_{L, R}=a^{-3 / 2} \sqrt{\frac{\pi}{4 H}}\left[J_{3 / 2}\left(\frac{k}{a H}\right)-i J_{-3 / 2}\left(\frac{k}{a H}\right)\right] \text { for } \frac{k}{a H} \gtrsim 1, \\
w_{L, R}=\frac{i}{\sqrt{2 k}}\left(\frac{H}{k}\right)\left[1+\frac{i}{3}\left(\frac{k}{a H}\right)^{3}\right] \simeq \frac{i}{\sqrt{2 k}}\left(\frac{H}{k}\right) \text { for } \frac{k}{a H} \ll 1 \ll \frac{3 H}{M}, \\
w_{L, R}=a^{-3 / 2} \sqrt{\frac{\pi}{4 H}}\left[i\left(\frac{a H}{k}\right)^{3 / 2}\left(\frac{3 H}{M}\right)^{1 / 2} J_{1 / 2}\left(\frac{M}{3 H}\right)+\right. \\
\left.+\frac{1}{3}\left(\frac{k}{a H}\right)^{3 / 2}\left(\frac{M}{3 H}\right)^{1 / 2} J_{-1 / 2}\left(\frac{M}{3 H}\right)\right] \text { for } \frac{3 H}{M} \lesssim 1,
\end{gathered}
$$

where we have used that

$$
\left(\frac{2 a_{k} H}{k}\right)^{4}=\frac{16}{3}\left(\frac{a H}{k}\right)^{3}\left(\frac{3 H}{M}\right)=\text { constant }
$$

as obtained by Eqs. (21) and (51). Eq. (55) corresponds to both the Eqs. (49) and (50) after the matching, as they are both of the form $w_{L, R}=C_{1}+C_{2} a^{-3}$, with $C_{i}$ being constants, which are identified in the two regimes by the matching. Calculating the power spectrum using Eq. (55), one finds the result shown in Eq. (27). This demonstrates

that the validity of this result extends beyond the $r \ll 1$ regime of the previous section, to the region with $r \gg 1$ as long as $M \ll H$.

The above analytic approximation is remarkably accurate as shown by comparison with the solution of Eq. (43) obtained numerically (see Fig. 2). The reason is that the physical scale $k / a$ evolves over an exponentially large range of values, whereas the approximation might be challenged only over a couple of orders of magnitude around the switch-over value $k / a_{k}=M_{k}$ (i.e. $r=1$ ).

\section{B. The longitudinal component}

Let us concentrate now on the longitudinal mode function. For the choice in Eq, (42), the equation of motion for the longitudinal mode function in Eq. (29) becomes

$$
\ddot{w}_{\|}+\left(3+\frac{8}{1+r^{2}}\right) H \dot{w}_{\|}+\left[\frac{24}{1+r^{2}} H^{2}+\left(\frac{k}{a}\right)^{2}\left(1+r^{2}\right)\right] w_{\|}=0 \text {. }
$$


The generic solution to the above can be obtained when $r \not 1$ and $r \not r_{c}$ (cf. Eq. (62)). Indeed, one finds

$$
\begin{gathered}
w_{\|}=a^{-11 / 2}\left[c_{3} J_{5 / 2}\left(\frac{k}{a H}\right)+c_{4} J_{-5 / 2}\left(\frac{k}{a H}\right)\right] \text { for } r \ll 1, \\
w_{\|}=a^{-3 / 2}\left[c_{3}^{\prime} J_{3 / 8}\left(\sqrt{\frac{3}{2}} \frac{1}{r}\right)+c_{4}^{\prime} J_{-3 / 8}\left(\sqrt{\frac{3}{2}} \frac{1}{r}\right)\right] \text { for } 1 \ll r \ll r_{c}, \\
w_{\|}=a^{-3 / 2}\left[\hat{c}_{3} J_{1 / 2}\left(\frac{M}{3 H}\right)+\hat{c}_{4} J_{-1 / 2}\left(\frac{M}{3 H}\right)\right] \text { for } r \gg r_{c},
\end{gathered}
$$

where $c_{i}, c_{i}^{\prime}$ and $\hat{c}_{i}$ are integration constants and $r_{c}$ is determined by the condition

$$
r_{c} \equiv 2 \sqrt{6} \frac{H}{M_{c}}
$$

which shows that $r_{c} \gg 1$ (considering Eq. (20)). The above condition corresponds to the moment when the first and last terms in the square brackets in Eq. (58) become comparable. ${ }^{4}$ Note that Eq. (61) looks the same as Eq. (45). This is because Eqs. (43) and (58) become identical for $r \gg r_{c}$.

In this case we need to do two matchings of $w_{\|}$and $\dot{w}_{\|}$; at $r=1$ and $r=r_{c}$. Let us perform the matching at $r_{c}$ first. In the vicinity of $r_{c}$ Eqs. (60) and (61) can be written respectively as

$$
\begin{gathered}
w_{\|}=a^{-3 / 2}\left[\frac{c_{3}^{\prime}}{\Gamma(11 / 8)}\left(\sqrt{\frac{3}{8}} \frac{1}{r}\right)^{3 / 8}+\frac{c_{4}^{\prime}}{\Gamma(5 / 8)}\left(\sqrt{\frac{8}{3}} r\right)^{3 / 8}\right] \text { for } r \lesssim r_{c} \quad \text { and } \\
w_{\|}=a^{-3 / 2} \sqrt{\frac{2}{\pi}}\left[\hat{c}_{3}\left(\frac{M}{3 H}\right)^{1 / 2}+\hat{c}_{4}\left(\frac{3 H}{M}\right)^{1 / 2}\right] \text { for } r \gtrsim r_{c} .
\end{gathered}
$$

Both the above are of the form $w_{\|}=C_{1}+C_{2} a^{-3}$, which means that they can be readily matched. Indeed, after matching one finds

$$
c_{3}^{\prime}=4 \sqrt{\frac{3}{\pi}}\left(\frac{8}{3}\right)^{3 / 16} \Gamma(11 / 8)\left(\frac{2 a_{k} H}{k}\right)^{1 / 2} \hat{c}_{4} \quad \text { and } \quad c_{4}^{\prime}=\frac{1}{3} \sqrt{\frac{3}{\pi}}\left(\frac{3}{8}\right)^{3 / 16} \Gamma(5 / 8)\left(\frac{k}{2 a_{k} H}\right)^{1 / 2} \hat{c}_{3} .
$$

Now we can proceed similarly to the transverse case and write the solutions in the vicinity of the $r=1$ switch-over as

$$
\begin{gathered}
w_{\|}=a^{-11 / 2} \sqrt{\frac{2}{\pi}}\left[\frac{1}{15} c_{3}\left(\frac{k}{a H}\right)^{5 / 2}+3 c_{4}\left(\frac{a H}{k}\right)^{5 / 2}\right] \text { for } r \lesssim 1 \quad \text { and } \\
w_{\|}=a^{-3 / 2} \sqrt{\frac{2}{\pi}}\left[\hat{c}_{3}\left(\frac{M}{3 H}\right)^{1 / 2}+\hat{c}_{4}\left(\frac{3 H}{M}\right)^{1 / 2}\right] \text { for } r>1 .
\end{gathered}
$$

Note that Eq. (67) incorporates both Eqs. (63) and (64), by virtue of the matching in Eq. (65).

As in the transverse case, to approximate the complete solution, we perform a matching at the $r=1$ switch-over of both the mode function $w_{\|}$and its time-derivative $\dot{w}_{\|}$, connecting thereby the $\hat{c}_{i}$ integration constants with the $c_{i}$. After matching we obtain

$$
\hat{c}_{3}=-\frac{4 \sqrt{3}}{9} a_{k}^{-4}\left(\frac{k}{2 a_{k} H}\right)^{2} c_{3} \quad \text { and } \quad \hat{c}_{4}=\sqrt{3} a_{k}^{-4}\left(\frac{2 a_{k} H}{k}\right)^{2}\left[\frac{1}{4} c_{4}+\frac{64}{135}\left(\frac{k}{2 a_{k} H}\right)^{5} c_{3}\right] .
$$

\footnotetext{
4 To visualise the physical interpretation of $r_{c}$ consider the range $[k / a, H]$, whose lower limit reduces in time. The effective mass of the physical vector field grows in time as $M \propto a^{3}$. When it becomes comparable to $k / a$ we have $r=1$ and $a=a_{k}$. After this moment, $M$ enters into the region in question and continues to grow approaching $H$. The moment where $r=r_{c}$ corresponds to when $M$ crosses the geometric mean of the region, i.e. $M=\sqrt{H(k / a)}$.
} 
To evaluate the $c_{i}$ we need to match the solution in Eq. (59) with the vacuum condition in Eq. (31). Thus, we obtain

$$
c_{3}=-\frac{i}{2} a_{k}^{4} \sqrt{\frac{\pi}{H}} \quad \text { and } \quad c_{4}=-\frac{1}{2} a_{k}^{4} \sqrt{\frac{\pi}{H}} .
$$

Hence, the analytic expression for the longitudinal mode function can be written as

$$
\begin{gathered}
w_{\|}=-i a^{-9 / 2} \sqrt{\frac{\pi}{4 H}}\left(\frac{a H}{k}\right)^{2}\left(\frac{H}{M}\right)\left[J_{5 / 2}\left(\frac{k}{a H}\right)-i J_{-5 / 2}\left(\frac{k}{a H}\right)\right] \text { for } \frac{k}{a H} \gtrsim 1, \\
w_{\|} \simeq-\frac{3 a_{k}^{4}}{\sqrt{2 H}}\left(\frac{H}{k}\right)^{5 / 2} a^{-3}=-\frac{1}{\sqrt{2 H}}\left(\frac{H}{k}\right)^{3 / 2}\left(\frac{3 H}{M}\right) \text { for } \frac{k}{a H} \ll 1 \ll \frac{3 H}{M}, \\
w_{\|}=a^{-3 / 2} \sqrt{\frac{\pi}{4 H}}\left[\frac{i}{3}\left(\frac{k}{a H}\right)^{3 / 2}\left(\frac{M}{3 H}\right)^{1 / 2} J_{1 / 2}\left(\frac{M}{3 H}\right)-\right. \\
\left.-\left(\frac{a H}{k}\right)^{3 / 2}\left(\frac{3 H}{M}\right)^{1 / 2} J_{-1 / 2}\left(\frac{M}{3 H}\right)\right] \text { for } \frac{3 H}{M} \lesssim 1,
\end{gathered}
$$

In the above, Eq. (71) corresponds to both Eqs. (70) and (72). This is because both equations, in the vicinity of the $r=1$ switch-over, are of the form $w_{\|}=C a^{-3}$, with $C$ a constant (see Eqs. (66) and (67), the latter also incorporating Eq. (60) in the limit $r>1$ ). This corresponds to the growing mode of Eq. (70) and the decaying mode of Eq. (72), which is dominant near $a \sim a_{k}$. It can also be shown that this mode remains dominant until $M \sim H$, i.e. beyond the $r \sim r_{c}$ switch-over. Calculating the power spectrum using Eq. (71), one finds the result shown in Eq. (37). As with the transverse case, this demonstrates that the validity of this result extends beyond the $r \ll 1$ regime of the previous section, to the region with $r \gg r_{c}$ as long as $M \ll H$. As was the case of the transverse modes, the above analytic approximation is remarkably accurate which is demonstrated by comparison with the solution of Eq. (58) obtained numerically (see Fig. 2).

\section{The power spectra}

In the previous section we found that the transverse and the longitudinal mode functions evolve in a similar manner. Indeed, the functions cease oscillating after horizon exit and until $M \sim H$. During this period they satisfy an equation of the form (cf. Eqs. (50) and (67))

$$
w_{\lambda}=a^{-3 / 2} \sqrt{\frac{2}{\pi}}\left[\hat{c}_{A}\left(\frac{M}{3 H}\right)^{1 / 2}+\hat{c}_{B}\left(\frac{3 H}{M}\right)^{1 / 2}\right] \quad \text { for } \quad M, \frac{k}{a} \ll H,
$$

where the $\hat{c}_{i}$ are appropriate constants. The above expression is of the form $w_{\lambda}=C_{A}+C_{B} a^{-3}$, with $C_{i}$ constants.

However, there is a crucial difference between the transverse and longitudinal modes. In the case of $w_{L, R}, \hat{c}_{A}$ and $\hat{c}_{B}$ in Eq. (73) are identified with $\hat{c}_{1}$ and $\hat{c}_{2}$ respectively, which are shown in Eq. (52). Using these values it can be readily confirmed that the dominant term in Eq. (73) in the regime $M, k / a \ll H$ is the "growing" mode: $w_{L, R}=$ constant. In contrast, in the case of $w_{\|}, \hat{c}_{A}$ and $\hat{c}_{B}$ in Eq. (173) are identified with $\hat{c}_{3}$ and $\hat{c}_{4}$ respectively, which are shown in Eq. (68). Using these values it can be readily confirmed that the dominant term in Eq. (73) in the regime $M, k / a \ll H$ is the decaying mode: $w_{\|} \propto a^{-3}$ (by virtue of the condition $M / H \ll 1 \ll a H / k$ ).

This difference in scaling is reflected, of course, on the power spectra, as already shown in Eqs. (27) and (37), which, as argued above, are valid in the regime where $M, k / a \ll H$. Thus, the typical value for the vector field perturbations in the regime in question scales as

$$
\delta W_{L, R}=\sqrt{\mathcal{P}_{+}}=\frac{H}{2 \pi}=\text { constant } \quad \text { and } \quad \delta W_{\|}=\sqrt{\mathcal{P}_{\|}}=\frac{3 H}{M} \frac{H}{2 \pi} \propto a^{-3},
$$

where $\delta W_{\lambda} \equiv\left|\delta \boldsymbol{W}_{\lambda}\right|$ and

$$
\mathcal{P}_{+} \equiv \frac{1}{2}\left(\mathcal{P}_{L}+\mathcal{P}_{R}\right)=\mathcal{P}_{L, R}
$$

because $\mathcal{P}_{L}=\mathcal{P}_{R}$ as the theory is parity invariant. 


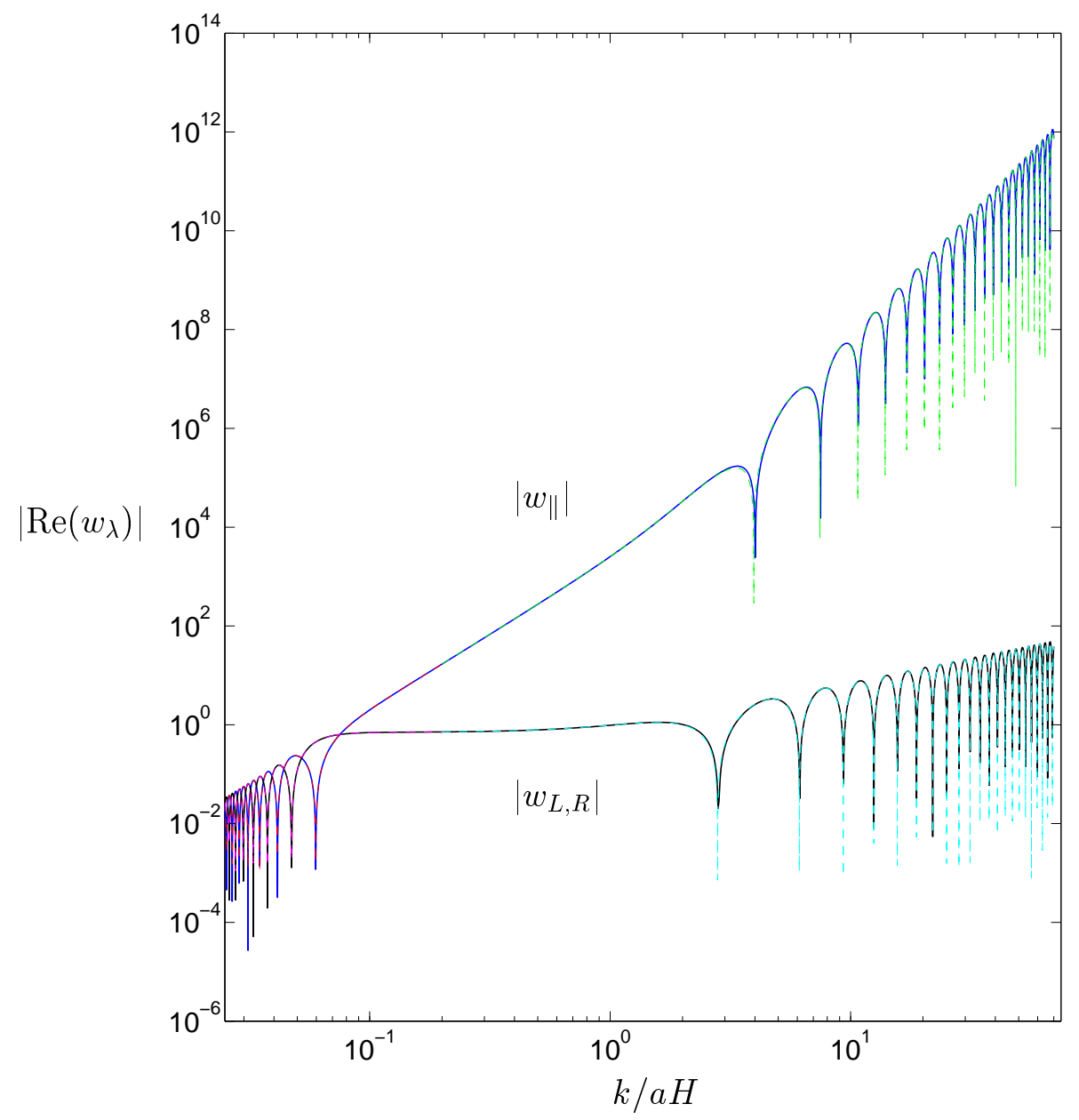

FIG. 2: Log-log plot of the evolution of the real part of the longitudinal (upper) and transverse (lower) mode functions $w_{\lambda}$ with $\lambda=\|, L, R$ of the vector field perturbations at a given, fixed $k$, in terms of the physical momentum scale $k / a H$ (weighted

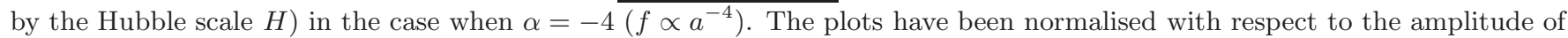
the transverse mode functions at horizon crossing $(k / a H=1)$. The solid lines depict the numerical solutions of the equations of motion in Eqs. (43) and (58), while the dashed lines depict that corresponding analytic approximations in Eqs. (54) - (56) and (70) - (72) respectively. The precision of the approximation is remarkable as the difference can hardly be seen. Notice that there are clearly three regimes for the evolution of the mode functions: First, when subhorizon, they undergo oscillations until horizon crossing $(k / a H \sim 1)$, when they enter a power-law regime, which eventually is terminated by another phase of oscillations, when the vector field becomes heavy $(M \gtrsim 3 H)$. The longitudinal mode function, when superhorizon, scales as $w_{\|} \propto a^{-3}$ before oscillations, in agreement with Eq. (71). In contrast, the transverse mode function remains constant with respect to $a$, as suggested by Eq. (55). Both mode functions oscillate with amplitude $\left\|w_{\lambda}\right\| \propto a^{-3}$ when the vector field becomes heavy, cf. Eq. (82). 
Now, let us find out how the perturbations behave when $M \sim H$. Eqs. (56) and (72) can be written as

$$
\begin{aligned}
2 \sqrt{\frac{H}{\pi}}\left(\frac{k}{H}\right)^{3 / 2} w_{L, R} & =\frac{i}{\sqrt{z}} J_{1 / 2}(z)+\frac{1}{3} x^{3} \sqrt{z} J_{-1 / 2}(z) \quad \text { and } \\
2 \sqrt{\frac{H}{\pi}}\left(\frac{k}{H}\right)^{3 / 2} w_{\|} & =\frac{1}{3} x^{3} \sqrt{z} J_{1 / 2}(z)-\frac{1}{\sqrt{z}} J_{-1 / 2}(z),
\end{aligned}
$$

where we defined

$$
z \equiv \frac{M}{3 H} \propto a^{3} \quad \text { and } \quad x \equiv \frac{k}{a H} \ll 1 .
$$

When $z \gtrsim 1$, for the amplitudes of the oscillating Bessel functions we have $\left\|J_{\nu}(z)\right\| \approx\left\|J_{-\nu}(z)\right\|$. Using this and also that $x \ll 1$ for superhorizon perturbations, we obtain

$$
\left.\begin{array}{rl}
2 \sqrt{\frac{H}{\pi}}\left(\frac{k}{H}\right)^{3 / 2} w_{L, R} & \simeq \frac{i}{\sqrt{z}} J_{1 / 2}(z) \\
2 \sqrt{\frac{H}{\pi}}\left(\frac{k}{H}\right)^{3 / 2} w_{\|} & \simeq-\frac{1}{\sqrt{z}} J_{-1 / 2}(z)
\end{array}\right\} \Rightarrow\left\|w_{L, R}\right\| \approx\left\|w_{\|}\right\| \quad \text { for } z \gtrsim 1 .
$$

Indeed, when $z \gg 1$ the mode functions approach

$$
w_{L, R}=\frac{i}{\sqrt{2 H}}\left(\frac{H}{k}\right)^{3 / 2} \frac{\sin z}{z} \quad \text { and } \quad w_{\|}=-\frac{1}{\sqrt{2 H}}\left(\frac{H}{k}\right)^{3 / 2} \frac{\cos z}{z} \quad \text { for } z \gg 1,
$$

where we used that $\lim _{z \rightarrow+\infty} J_{\nu}(z)=\sqrt{\frac{2}{\pi z}} \cos \left[z-\frac{\pi}{2}\left(\nu-\frac{1}{2}\right)\right]$. Since $z \gg 1$, the frequency of oscillations is very large compared to the expansion rate $H$. This means that it makes sense to use the average values of the power spectra over many oscillations. In view of Eq. (80), we find

$$
\overline{\mathcal{P}_{+}}=\overline{\mathcal{P}_{\|}}=\frac{1}{2 z^{2}}\left(\frac{H}{2 \pi}\right)^{2} .
$$

Thus, the typical value for the vector field perturbations in this regime is

$$
\delta W_{L, R}=\delta W_{\|}=\frac{1}{\sqrt{2}} \frac{3 H}{M} \frac{H}{2 \pi} \propto a^{-3},
$$

where we used $\delta W_{\lambda}=\sqrt{\overline{\mathcal{P}_{\lambda}}}$, where $\lambda=+, \|$ and we have $\delta W_{L}=\delta W_{R} \equiv \delta W_{+}$. We have found that, for $M \gtrsim H$, the mode functions of the transverse and longitudinal components oscillate rapidly with the same amplitude, so that the components of the typical perturbations of the vector field are equal.

\section{Deviations from scale invariance}

So far we have discussed the case of exact scale invariance for both the transverse and longitudinal spectra. A small deviation from scale invariance is favoured at the moment by the observations. Such a deviation can be achieved by perturbing the scalings of $f$ and $m$. Indeed, introducing the perturbations

$$
\alpha=-4\left(1+\epsilon_{f}\right) \quad \text { and } \quad \beta=1+\epsilon_{m},
$$

one can repeat the calculations to find the scale invariance of the spectra. Parameterising this scale invariance in the usual manner, i.e.

$$
\mathcal{P}_{\lambda} \propto k^{n_{\lambda}-1}
$$

with $\lambda=\|,+$, we have found the following expressions for the spectral indexes:

$$
n_{\|}-1=-\left(\epsilon_{f}+2 \epsilon_{m}\right) \quad \text { and } \quad n_{+}-1=-\frac{1}{4}\left(18 \epsilon_{f}+\epsilon_{m}\right) .
$$

We see that, for positive $\epsilon_{i}$ 's, both the spectra are red as required by the observations. The spectral indexes are identified if $\epsilon_{m} \approx 2 \epsilon_{f}$. In this case $n_{\lambda} \simeq 0.96$ is obtained with $\epsilon_{f} \simeq 8 \times 10^{-3}$. Deviation from scale invariance can be also produced by considering that inflation is not exactly de Sitter, i.e. $\varepsilon \equiv-\dot{H} / H^{2} \neq 0$. According to the usual curvaton result, this would introduce a red tilt in the spectral indexes of magnitude $\delta n_{\lambda}=-2 \varepsilon$. Thus, $\varepsilon \approx 0.02$ may be enough to satisfy the observations, for $(\alpha, \beta)=(-4,1)$. 


\section{CASE: $f \propto a^{2} \& m \propto a$}

Here we study the other possibility which allows scale invariant spectra for all the components of the perturbations of our vector field. Hence, in this section we assume the values

$$
\alpha=2 \quad \& \quad \beta=1
$$

thus making $f \propto a^{2}$ and $M=$ constant. The condition that the physical vector field is effectively massless at the time of horizon exit in Eq. (20) suggests that $M / H \ll 1$ at all times when the scaling above holds. Using this condition and scaling we can calculate the power spectra for all the components of the superhorizon vector field perturbations generated by the particle production process.

\section{A. The transverse components}

For the choice of scaling above, the equation of motion for the transverse mode functions in Eq. (18) becomes

$$
\ddot{w}_{L, R}+3 H \dot{w}_{L, R}+\left(\frac{k}{a}\right)^{2}\left(1+r^{2}\right) w_{L, R}=0,
$$

which has the same form as the equation of motion for the $\alpha=-4$ case. However, because $M$ is constant we may obtain an exact solution to the equation of motion above

$$
w_{L, R}=-a^{-3 / 2} \sqrt{\frac{\pi}{4 H}}\left[J_{\tilde{\nu}}\left(\frac{k}{a H}\right)-i J_{-\tilde{\nu}}\left(\frac{k}{a H}\right)\right]
$$

where

$$
\tilde{\nu} \equiv \frac{1}{2} \sqrt{9-4\left(\frac{M}{H}\right)^{2}} \simeq \frac{3}{2}
$$

As before, we have used the Bunch-Davis vacuum boundary condition in Eq. (22) to obtain the constants of integration. Taking the limit of this solution at late times (superhorizon scales) we can calculate the power spectrum

$$
\mathcal{P}_{+}=\left(\frac{H}{2 \pi}\right)^{2},
$$

which is in agreement with Eq. (27) and we considered $\tilde{\nu} \simeq \frac{3}{2}$. However if we consider the next level of perturbation $\tilde{\nu} \simeq \frac{3}{2}-\frac{1}{3}(M / H)^{2}$, then we get a slight deviation from scale invariance $\mathcal{P}_{+} \propto k^{n_{+}-1}$ where

$$
n_{+}-1=\frac{2}{3}\left(\frac{M}{H}\right)^{2} \equiv 2 \eta_{A} .
$$

As evident, this deviation from scale invariance gives rise to a blue tilt on the spectrum. Alternatively we may perturb the scaling slightly as

$$
\alpha=2\left(1+\epsilon_{f}\right) \quad \text { and } \quad \beta=1+\epsilon_{m},
$$

in analogy with Eq. (83). If $\epsilon_{f, m}>M / H$ then the scale dependence becomes

$$
n_{+}-1=-\frac{8}{3} \epsilon_{f},
$$

which allows a red spectrum if $\epsilon_{f}<0$. 


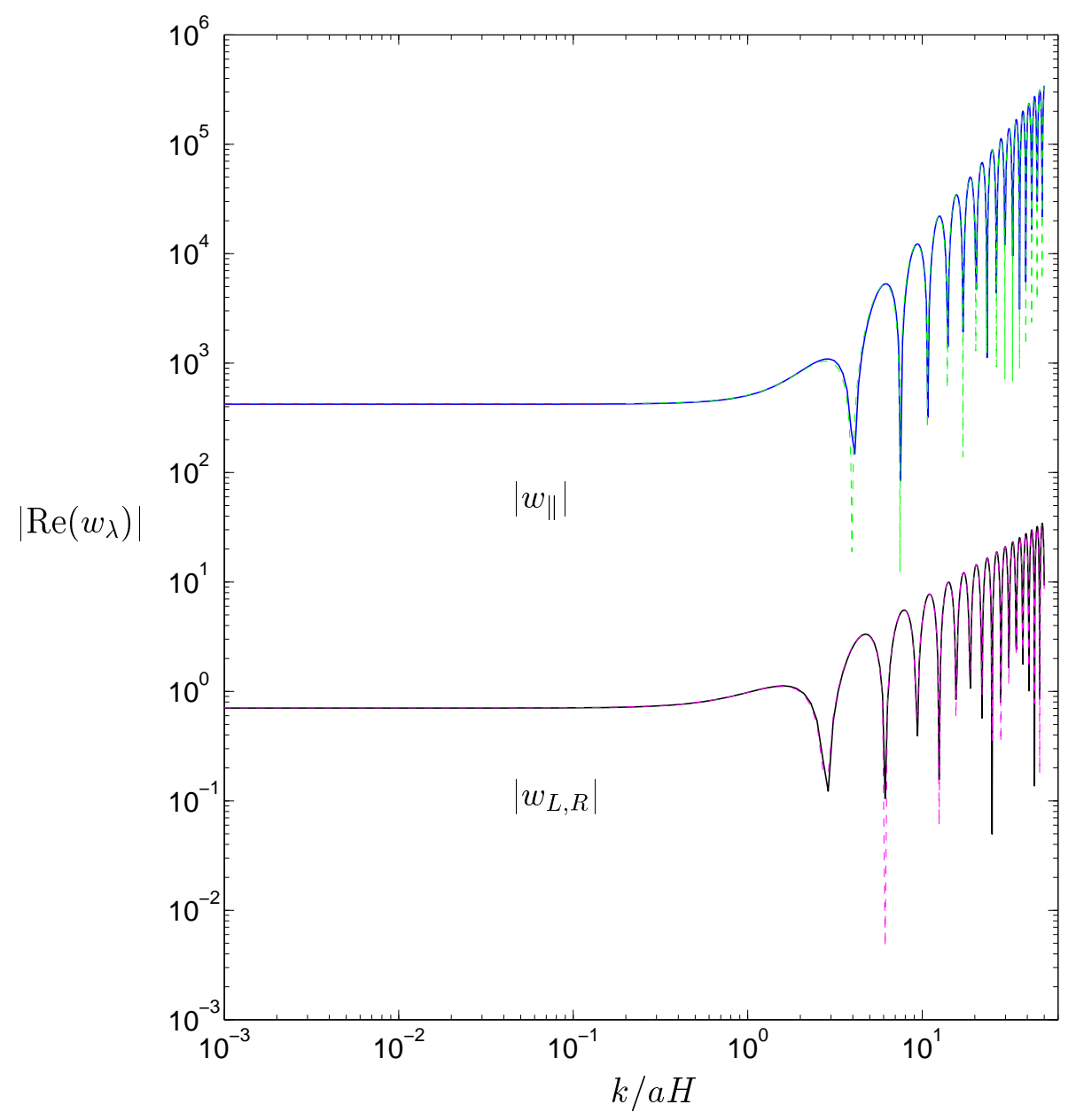

FIG. 3: Log-log plot of the evolution of the real part of the longitudinal (upper) and transverse (lower) mode functions $w_{\lambda}$ with $\lambda=\|, L, R$ of the vector field perturbations at a given, fixed $k$, in terms of the physical momentum scale $k / a H$ (weighted

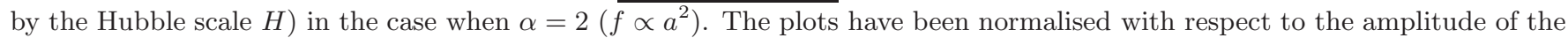
transverse mode function at horizon crossing $(k / a H=1)$. The solid lines depict the numerical solutions of the equations of motion in Eqs. (87) and (94), while the dashed lines depict the corresponding analytic solution in Eq. (88) and the approximation in Eqs. (97) and (98) respectively. The precision of the approximation is remarkable as the difference can hardly be seen. Notice that there are clearly two regimes for the evolution of the mode functions: First, when subhorizon, they undergo oscillations until horizon crossing $(k / a H \sim 1)$, when they enter a power-law regime. Both mode functions, when superhorizon, remain constant with respect to $a$ in agreement, e.g. with Eq. (98) for $w_{\|}$. Because $M \ll H$, we have $w_{\|} \gg w_{L, R}$. 


\section{B. The longitudinal component}

Let us concentrate now on the longitudinal mode function. For the choice of scaling in Eq. (86), the equation of motion for the longitudinal mode function in Eq. (29) becomes

$$
\ddot{w}_{\|}+\left(3+\frac{2}{1+r^{2}}\right) H \dot{w}_{\|}+\left[\left(\frac{k}{a}\right)^{2}\left(1+r^{2}\right)\right] w_{\|}=0 .
$$

Using the same technique as in the previous case we find exact solutions in two regimes

$$
\begin{array}{r}
w_{\|}=a^{-5 / 2}\left[c_{5} J_{-5 / 2}\left(\frac{k}{a H}\right)+c_{6} J_{5 / 2}\left(\frac{k}{a H}\right)\right] \text { for } r \ll 1 \quad \text { and } \\
w_{\|}=\hat{c}_{5}+\hat{c}_{6} a^{-3} \text { for } r \gg 1
\end{array}
$$

where $c_{i}, \hat{c}_{i}$ are integration constants. Again we have used $M / H=$ constant $\ll 1$. As in the previous case, using the vacuum boundary condition in Eq. (31), we can find the values of $c_{5,6}$. Then, matching the two solutions and their first derivatives at $k / a H=M / H$ (i.e. $r=1$ ) we can find $\hat{c}_{5,6}$ in the regime $r \gg 1$. Thus, the solutions become

$$
\begin{gathered}
w_{\|}=-a^{-5 / 2} \frac{k}{M} \sqrt{\frac{\pi}{4 H}}\left[J_{-5 / 2}\left(\frac{k}{a H}\right)+i J_{5 / 2}\left(\frac{k}{a H}\right)\right] \text { for } \frac{k}{a H} \gtrsim 1, \\
w_{\|}=\frac{i}{\sqrt{2 k}} \frac{H^{2}}{M k}\left\{\left[3 i+\frac{2}{45}\left(\frac{M}{H}\right)^{5}\right]-\frac{1}{9}\left(\frac{M}{H}\right)^{2}\left(\frac{k}{a H}\right)^{3}\right\} \simeq-\frac{3}{\sqrt{2 k}}\left(\frac{H}{M}\right)\left(\frac{H}{k}\right) \text { for } \frac{k}{a H} \ll 1 \ll \frac{3 H}{M} .
\end{gathered}
$$

As in the previous section, the above analytic approximation is remarkably accurate, as demonstrated in Fig. 3.

Using Eq. (98), we can calculate the power spectrum at arbitrary late times

$$
\mathcal{P}_{\|}=9\left(\frac{H}{M}\right)^{2}\left(\frac{H}{2 \pi}\right)^{2},
$$

which is in agreement with Eq. (37). Since in this case, we have $M / H=$ constant $\ll 1$, we see that the longitudinal power spectrum is constant, in contrast to the $\alpha=-4$ case. Also, we see that $\mathcal{P}_{\|} \gg \mathcal{P}_{+}$.

Once again if we introduce perturbations to the scaling as in Eq. (92) we can find a slight deviation in the scale dependence

$$
n_{\|}-1=2\left(\epsilon_{f}-\epsilon_{m}\right) .
$$

\section{STATISTICAL ANISOTROPY AND NON-GAUSSIANITY}

The theory studied in this paper has two clear advantages. First we can obtain a completely isotropic perturbation spectrum for the vector field, which has previously never been achieved. As we discuss below, this means that we may consider vector fields as dominating the total energy density of the Universe when the curvature perturbation is formed. The second advantage is that we can also account for a small amount of statistical anisotropy in the curvature perturbation spectrum depending on when inflation ends, again by considering the vector field alone. We also demonstrate this in what follows. Finally, statistical anisotropy can also be present in a correlated manner in the bispectrum as well, which characterises the non-Gaussian features of the CMB temperature perturbations. In view of the forthcoming observations of the recently launched Planck satellite mission this is a particularly promising and timely result.

Let us begin with the generic treatment before focusing on cases of particular interest. As in Ref. [12], keeping only the leading term in the anisotropy, the power spectrum may be parameterised in the following fashion

$$
\mathcal{P}_{\zeta}(\mathbf{k})=\mathcal{P}_{\zeta}^{\text {iso }}(k)\left[1+g(\hat{\boldsymbol{d}} \cdot \hat{\boldsymbol{k}})^{2}\right],
$$


where $\mathcal{P}_{\zeta}^{\text {iso }}$ is the isotropic part of the power spectrum, $\hat{\boldsymbol{k}} \equiv \boldsymbol{k} / k$, and $\hat{\boldsymbol{d}}$ is a unit vector in some chosen direction. In the above the parameter $g$ quantifies the statistical anisotropy in the spectrum. In Ref. [12], it has been shown that, currently, the data suggest that $g \lesssim 0.3$ or so.

As shown in Ref. [22], when the anisotropy in the spectrum is due to the contribution of a vector field, then at tree level (first order) we have

$$
\hat{d}=\hat{N}_{A}
$$

where $\hat{\boldsymbol{N}}_{\boldsymbol{A}} \equiv \boldsymbol{N}_{\boldsymbol{A}} / N_{A}, N_{A} \equiv\left|\boldsymbol{N}_{\boldsymbol{A}}\right|$ and the components of $\boldsymbol{N}_{\boldsymbol{A}}$ are defined as

$$
N_{A}^{i} \equiv \frac{\partial N}{\partial W_{i}},
$$

with $N$ being the number of remaining e-folds of inflation. The vector $\boldsymbol{N}_{\boldsymbol{A}}$ quantifies (to first order) the contribution to the curvature perturbation $\zeta$ from the vector field only; as defined in the $\delta N$ formalism [23]. In this formalism the magnitude of anisotropy is characterised by [22]

$$
g \equiv N_{A}^{2} \frac{\mathcal{P}_{\|}-\mathcal{P}_{+}}{\mathcal{P}_{\zeta}^{\text {iso }}}
$$

and the isotropic part of the spectrum is

$$
\mathcal{P}_{\zeta}^{\text {iso }} \equiv N_{\phi}^{2} \mathcal{P}_{\phi}+N_{A}^{2} \mathcal{P}_{+}=N_{\phi}^{2} \mathcal{P}_{\phi}\left(1+\xi \frac{\mathcal{P}_{+}}{\mathcal{P}_{\phi}}\right)
$$

where we have defined

$$
\xi \equiv\left(\frac{N_{A}}{N_{\phi}}\right)^{2}
$$

and

$$
N_{\phi} \equiv \frac{\partial N}{\partial \phi}
$$

In the above $\phi$ is a scalar field which also contributes to the curvature perturbation. Its contribution is necessary if the anisotropy due to the vector field is excessive. In this case the vector field can only generate a subdominant contribution to $\zeta$, with the dominant contribution due to some other source, e.g. the scalar field $\phi$. If, however, the anisotropy due to the vector field is within the observational bounds then one can dispense with the scalar field. Thus, if this is the case, we can assume that the scalar field modulation of $N$ is negligible so that $N_{\phi} \rightarrow 0$. Therefore, dispensing with the scalar field contribution is equivalent to $\xi \rightarrow \infty$.

Now, from Eqs. (105) and (106) we can write the anisotropy parameter in Eq. (101) as

$$
g=\xi \frac{\mathcal{P}_{\|}-\mathcal{P}_{+}}{\mathcal{P}_{\phi}+\xi \mathcal{P}_{+}}
$$

Hence, we can calculate the level of statistical anisotropy in the power spectrum.

How do the above translate in the case of the vector curvaton mechanism? As calculated in Ref. [22], the tree-level contribution to the curvature perturbation spectrum from the vector curvaton field is

$$
\mathcal{P}_{\zeta_{A}}(\boldsymbol{k})=\frac{4}{9} \frac{\hat{\Omega}_{A}^{2}}{W^{2}}\left[\mathcal{P}_{+}+\left(\mathcal{P}_{\|}-\mathcal{P}_{+}\right)(\hat{\boldsymbol{W}} \cdot \hat{\boldsymbol{k}})^{2}\right],
$$

where $\hat{\boldsymbol{W}} \equiv \boldsymbol{W} / W$ and $W \equiv|\boldsymbol{W}|$. In the above $\hat{\Omega}_{A}$ is defined as

$$
\hat{\Omega}_{A} \equiv \frac{3 \Omega_{A}}{4-\Omega_{A}} \sim \Omega_{A} \equiv \frac{\rho_{A}}{\rho},
$$

where $\rho_{A}$ is the density of the vector field. As in the case of the scalar curvaton paradigm, Eq. (109) should be evaluated at the time of decay of the curvaton field. 
As we have shown, in the case when $\alpha=-4$ and if $M \gtrsim H$ by the end of inflation, $\mathcal{P}_{+} \approx \mathcal{P}_{\|}$and statistical anisotropy in the spectrum can be very small; within the observational bounds. This means that the vector field can alone give rise to the observed curvature perturbation. Thus, if this is the case, we can dispense with the scalar field $\phi$ and we can write $\mathcal{P}_{\zeta}(\boldsymbol{k})=\mathcal{P}_{\zeta_{A}}(\boldsymbol{k})$. Then, from Eqs. (101), (105) and (109) we find

$$
\mathcal{P}_{\zeta}^{\text {iso }}=N_{A}^{2} \mathcal{P}_{+} \quad \text { with } \quad N_{A}=\frac{2}{3} \frac{\hat{\Omega}_{A}}{W}
$$

and also $g=\left(\mathcal{P}_{\|} / \mathcal{P}_{+}\right)-1$ which agrees with Eq. (108) in the limit $\xi \rightarrow \infty$.

As shown in Ref. [22], apart from statistical anisotropy in the spectrum of the curvature perturbations a vector field can give rise to statistical anisotropy in the bispectrum, which characterises the non-Gaussian features of the cosmological perturbations. Non-Gaussianity is an observable of great importance as it can be a powerful discriminator between mechanisms for the generation of the curvature perturbation. It was shown recently in Ref. [35] that the statistical anisotropy in the spectrum and bispectrum are correlated if they are generated by a vector field. Such correlation can be a signature prediction of the vector curvaton scenario. If it is observed it would be direct evidence for a vector field contribution to the curvature perturbation.

Non-Gaussianity expresses a non-vanishing 3-point correlator, or equivalently a non-zero bispectrum. The bispectrum is a function of three $\boldsymbol{k}_{1,2,3}$ vectors which may be chosen arbitrarily. However we will focus on the two standard configurations; the equilateral and squeezed (or local) configurations where the magnitudes satisfy $k_{1}=k_{2}=k_{3}$ and $k_{1} \simeq k_{2} \gg k_{3}$ respectively. It was shown in Ref. [35] that the expressions for $f_{\mathrm{NL}}$, the non-linearity parameter characterising non-Gaussianity, for a scale invariant power spectrum in the equilateral and local configurations respectively are given by

$$
\frac{6}{5} f_{\mathrm{NL}}^{\text {equil }}=\xi^{2} \mathcal{P}_{+}^{2} \frac{3}{2 \hat{\Omega}_{A}} \frac{\left(1+\frac{1}{2} q^{2}\right)+\left[p+\frac{1}{8}\left(p^{2}-2 q^{2}\right)\right] \hat{W}_{\perp}^{2}}{\left(\mathcal{P}_{\phi}+\xi \mathcal{P}_{+}\right)^{2}}
$$

and

$$
\frac{6}{5} f_{\mathrm{NL}}^{\text {local }}=\xi^{2} \mathcal{P}_{+}^{2} \frac{3}{2 \hat{\Omega}_{A}} \frac{1+p \hat{W}_{\perp}^{2}}{\left(\mathcal{P}_{\phi}+\xi \mathcal{P}_{+}\right)^{2}},
$$

where $\hat{W}_{\perp} \equiv\left|\hat{\boldsymbol{W}}_{\perp}\right|$, with $\hat{\boldsymbol{W}}_{\perp}$ being the projection of the unit vector $\hat{\boldsymbol{W}}$ to the plane defined by the three $\boldsymbol{k}_{1,2,3}$ vectors.

We have also defined

$$
p \equiv \frac{\mathcal{P}_{\|}-\mathcal{P}_{+}}{\mathcal{P}_{+}} \quad \text { and } \quad q \equiv \frac{\mathcal{P}_{-}}{\mathcal{P}_{+}},
$$

with

$$
\mathcal{P}_{-} \equiv \frac{1}{2}\left(\mathcal{P}_{L}-\mathcal{P}_{R}\right)=0
$$

i.e. $q=0$ since our theory is parity invariant.

In the following, to calculate $f_{\mathrm{NL}}$, we assume that $\mathcal{P}_{\phi}$ is due to a light scalar field, in which case

$$
\mathcal{P}_{\phi}=\left(\frac{H}{2 \pi}\right)^{2} .
$$

The curvature perturbation in Eq. (101) and the vector field contribution to it in Eq. (109) should be calculated at the time of the decay of the vector field, as in the scalar curvaton scenario. In contrast, the spectra of the vector field perturbations $\mathcal{P}_{\lambda}$ are calculated either at the onset of the vector field oscillations or at the end of the scaling of $f(a)$ and $m(a)$; whichever occurs latest, regardless of whether this moment is during or after inflation. Therefore, at that time, $f=1$ and $M=\hat{m}$, where $\hat{m}=$ constant is the final (vacuum) value of the vector field mass.

As we have shown, the evolution of the components of the vector field perturbations goes through the following stages. When subhorizon, the perturbations are oscillating (starting of as quantum fluctuations). After horizon crossing, they follow a power law evolution of the form $\delta W_{\lambda}=C_{1}+C_{2} a^{-3}$, where $C_{i}$ are constants. In the $\alpha=-4$ case the effective mass $M$ is increasing with time, which may allow the the vector field and its perturbations to begin oscillations (when $M \sim H$ ) before the end of the scaling behaviour of $f$ and $m$, i.e. before the end of inflation. In contrast, in the $\alpha=2$ case, since $M=$ constant $\ll H$, the perturbations remain in the power-law regime until the end of scaling. This suggests that, in this case, $\hat{m}<H$. Thus, when $\alpha=2$, the oscillations commence only after inflation, when $H(t)$ decreases enough such that $\hat{m} \sim H(t)$. In view of the above we can now calculate the statistical anisotropy in the spectrum and bispectrum of our model. 


\section{A. The power-law regime}

The curvature perturbation is formed in this period of evolution if $\hat{m} \lesssim H_{*}$, where $H_{*}$ denotes the Hubble scale during inflation. Then, from Eqs. (27) and (37) we have $\mathcal{P}_{\|}=\left(\frac{3 H_{*}}{\hat{m}}\right)^{2} \mathcal{P}_{+}$, which means that $\mathcal{P}_{\|} \gtrsim \mathcal{P}_{+}$. Therefore, our model gives

$$
p=\left(\frac{3 H_{*}}{\hat{m}}\right)^{2}-1 \quad \text { and } \quad g=\left(\frac{\xi}{1+\xi}\right)\left[\left(\frac{3 H_{*}}{\hat{m}}\right)^{2}-1\right]
$$

where $\xi$ is to be calculated at the decay of the vector field, which occurs after the onset of the vector field oscillations. The latter, in this case, has to occur after the end of inflation.

In this regime we also observe that $\mathcal{P}_{+}=\mathcal{P}_{\phi}$. Substituting Eq. (117) into Eqs. (112) and (113) (with $M \rightarrow \hat{m}$ ) we can now get expressions for the non-linearity parameter $f_{\mathrm{NL}}$ in terms of the anisotropy parameter $g$. We find

$$
\begin{aligned}
\frac{6}{5} f_{\mathrm{NL}}^{\text {equil }} & =\left(\frac{\xi}{1+\xi}\right)^{2} \frac{3}{2 \hat{\Omega}_{A}}\left(1+\left\{\frac{1}{8}\left[\left(\frac{3 H_{*}}{\hat{m}}\right)^{2}+3\right]^{2}-2\right\} \hat{W}_{\perp}^{2}\right) \\
& =\frac{g^{2}}{\left[\left(\frac{3 H_{*}}{\hat{m}}\right)^{2}-1\right]^{2}} \frac{3}{2 \hat{\Omega}_{A}}\left(1+\left\{\frac{1}{8}\left[\left(\frac{3 H_{*}}{\hat{m}}\right)^{2}+3\right]^{2}-2\right\} \hat{W}_{\perp}^{2}\right)
\end{aligned}
$$

and

$$
\begin{aligned}
\frac{6}{5} f_{\mathrm{NL}}^{\text {local }} & =\left(\frac{\xi}{1+\xi}\right)^{2} \frac{3}{2 \hat{\Omega}_{A}}\left\{1+\left[\left(\frac{3 H_{*}}{\hat{m}}\right)^{2}-1\right] \hat{W}_{\perp}^{2}\right\} \\
& =\frac{g^{2}}{\left[\left(\frac{3 H_{*}}{\hat{m}}\right)^{2}-1\right]^{2}} \frac{3}{2 \hat{\Omega}_{A}}\left\{1+\left[\left(\frac{3 H_{*}}{\hat{m}}\right)^{2}-1\right] \hat{W}_{\perp}^{2}\right\} .
\end{aligned}
$$

In analogy with Eq. (101) we can write

$$
f_{\mathrm{NL}}=f_{\mathrm{NL}}^{\mathrm{iso}}\left(1+\mathcal{G} \cdot \hat{W}_{\perp}^{2}\right)
$$

where $\mathcal{G}$ quantifies the statistical anisotropy in the bispectrum. It is evident that the direction of the statistical anisotropy in the bispectrum is correlated with the one in the spectrum (cf. Eq. (109)), since they are both determined by the direction of the unit vector $\hat{\boldsymbol{W}}$ exactly as in Ref. [35]. At the moment, observations do not provide any information about the value of $\mathcal{G}$.

\section{Highly anisotropic and subdominant limit}

Suppose that, at the end of scaling, $\mathcal{P}_{\|} \gg \mathcal{P}_{+}$. This is equivalent to $\left(H_{*} / \hat{m}\right)^{2} \gg 1$. In this limit we find $p=\left(3 H_{*} / \hat{m}\right)^{2} \gg 1$. Thus, Eq. (108) gives

$$
g=\left(\frac{\xi}{1+\xi}\right)\left(\frac{3 H_{*}}{\hat{m}}\right)^{2}
$$

Were we to dispense with the scalar field, i.e. if the vector field contribution to $\zeta$ were dominant, then $\xi \gg 1$ and the above would give $g=\left(3 H_{*} / \hat{m}\right)^{2} \gg 1$, which clearly violates the observational constraints. Therefore, the contribution of the vector field perturbations to the curvature perturbation must be subdominant to that of the scalar field. Thus, we must have $N_{A} \ll N_{\phi}$, which means $\xi \ll 1$. Similarly, the vector field contribution to the total energy density of the Universe has to be subdominant as well $\Omega_{A} \ll 1$. Hence, Eq. (110) suggests $\hat{\Omega}_{A} \rightarrow \frac{3}{4} \Omega_{A}$. Using this, for the case when $\left(H_{*} / \hat{m}\right)^{2} \gg 1$ we find

$$
\frac{6}{5} f_{\mathrm{NL}}^{\text {equil }} \simeq \frac{2 g^{2}}{\Omega_{A}}\left(\frac{\hat{m}}{3 H_{*}}\right)^{4}\left[1+\frac{1}{8}\left(\frac{3 H_{*}}{\hat{m}}\right)^{4} \hat{W}_{\perp}^{2}\right]
$$


and

$$
\frac{6}{5} f_{\mathrm{NL}}^{\text {local }} \simeq \frac{2 g^{2}}{\Omega_{A}}\left(\frac{\hat{m}}{3 H_{*}}\right)^{4}\left[1+\left(\frac{3 H_{*}}{\hat{m}}\right)^{2} \hat{W}_{\perp}^{2}\right] .
$$

From the above we see that, in this case, $f_{\mathrm{NL}} \propto g$, i.e. statistical anisotropy in the spectrum of the curvature perturbation would intensify the non-Gaussianity as is the case in Ref. [35]. Furthermore, by comparison with Eq. (120), we see that, for both equilateral and local configurations, for the isotropic part we have

$$
\frac{6}{5} f_{\mathrm{NL}}^{\mathrm{iso}} \simeq \frac{2 g^{2}}{\Omega_{A}}\left(\frac{\hat{m}}{3 H_{*}}\right)^{4}=\frac{2 \xi^{2}}{\Omega_{A}}
$$

which can, in principle, be substantial even if $\xi \ll 1$ because $\Omega_{A}$ can be very small. Notice however, that, in both configurations, $\mathcal{G} \gg 1$, by virtue of the condition $\hat{m}<H_{*}$. This means that, in this case, the statistical anisotropy in the bispectrum is dominant and would be readily detected if there is a confirmed detection of a non-zero $f_{\mathrm{NL}}$. Turning this around, if non-Gaussianity is not observed to be predominantly anisotropic this would rule out the $\hat{m}<H_{*}$ regime of this model.

From Eqs. (122) and (123) we readily obtain that the amplitudes of the modulated $f_{\mathrm{NL}}$ in both configurations are

$$
\frac{6}{5}\left\|f_{\mathrm{NL}}^{\text {local }}\right\|=2 \frac{g^{2}}{\Omega_{A}}\left(\frac{\hat{m}}{3 H_{*}}\right)^{2} \text { and } \quad \frac{6}{5}\left\|f_{\mathrm{NL}}^{\text {equil }}\right\|=\frac{1}{4} \frac{g^{2}}{\Omega_{A}}
$$

Hence, in general $\left\|f_{\mathrm{NL}}^{\text {local }}\right\|<\left\|f_{\mathrm{NL}}^{\text {equil }}\right\|$. This means that the observed upper bound $f_{\mathrm{NL}} \lesssim \mathcal{O}\left(10^{2}\right)$ [41] should be applied to the equilateral amplitude. Hence we obtain that $g^{2} / \Omega_{A} \lesssim 10^{2}$, which means that $f_{\mathrm{NL}}^{\text {iso }} \lesssim \mathcal{O}(1)$, since $\hat{m}<H_{*}$ in this regime.

Before moving on, it is important to stress that the above results are valid for $\alpha=-1 \pm 3$, i.e. for both $f \propto a^{-4}$ and $f \propto a^{2}$, as the condition $\hat{m}^{2} \ll H_{*}^{2}$ is possible in both cases. In fact, this is the only possibility for the case when $\alpha=2$.

\section{Almost isotropic and dominant limit}

In the case when $\alpha=-4, M$ is growing with time and the bound in Eq. (20) can be satisfied even with $\hat{m}$ not smaller than $H_{*}$. Therefore, in this case we can investigate the possibility that $\hat{m} \sim H_{*}$, which corresponds to the edge of the power-law regime (which is inaccessible in the $\alpha=2$ case).

Considering this regime we find $\mathcal{P}_{\|} \sim \mathcal{P}_{+}=\mathcal{P}_{\phi}$. Thus, in this case we find an almost isotropic curvature perturbation, which allows us to dispense with the scalar field and assume that the curvature perturbation is dominated by the vector field contribution. Hence, we can take $N_{A} \gg N_{\phi}$, i.e. $\xi \gg 1$. Then, from Eqs. (108), (114) and (117) we find

$$
\left(\frac{3 H_{*}}{\hat{m}}\right)^{2}-1=g=p=\frac{\delta \mathcal{P}}{\mathcal{P}_{+}}
$$

where $\delta \mathcal{P} \equiv \mathcal{P}_{\|}-\mathcal{P}_{+}$. Thus, if the fractional difference of the spectra is not excessive, the vector field might generate statistical anisotropy in the CMB within the observational bounds. Using the above, it is straightforward to show that

$$
\frac{6}{5} f_{\mathrm{NL}} \simeq \frac{3}{2 \hat{\Omega}_{A}}\left(1+g \hat{W}_{\perp}^{2}\right)
$$

in both the local and equilateral configurations. By comparison with Eq. (120) we see that, in this case, $\mathcal{G} \approx g$. Hence, statistical anisotropy is of the same magnitude in both the spectrum and bispectrum of the curvature perturbations. Therefore, observational constraints on $g$ in Ref. 12] suggest that $f_{\mathrm{NL}}$ may feature an angular modulation at a level as large as $30 \%$ or so.

In the isotropic and dominant limit when $\hat{m} \rightarrow 3 H_{*}$ we find that $g=0$ and

$$
f_{\mathrm{NL}}=\frac{5}{4 \hat{\Omega}_{A}} .
$$

As expected, this is equivalent to the scalar curvaton scenario [2]. Thus, in this case, substantial non-Gaussianity can be generated if the vector field decays before it dominates the Universe, with $\Omega_{A} \ll 1$. 


\section{B. The late-time oscillations regime}

As we have shown in Sec. IVC in the case when $\alpha=-4$, it is possible that $M$ grows much larger than $H$ before the end of scaling. This is indeed so if $\hat{m} \gg H_{*}$. Then, after $M \sim H$, the vector field perturbations undergo rapid oscillations, during which, $\overline{\mathcal{P}_{\|}}=\overline{\mathcal{P}_{+}}$as shown in Eq. (81). This means that $g=0$ and $p=0$ (cf. Eqs. (108) and (114) respectively), which results in an isotropic power spectrum. In this regime, from Eqs. (81) and (116), we also find that

$$
\overline{\mathcal{P}}_{\|,+}=\frac{1}{2}\left(\frac{3 H}{M}\right)^{2} \mathcal{P}_{\phi}
$$

Therefore Eqs. (112) and (113) reduce to

$$
\frac{6}{5} f_{\mathrm{NL}}=\xi^{2} \frac{3}{2 \hat{\Omega}_{A}}\left[2\left(\frac{\hat{m}}{3 H_{*}}\right)^{2}+\xi\right]^{-2},
$$

which is valid in both the local and equilateral configurations.

Since the vector field perturbations are isotropic we have no need of the scalar field contribution. As discussed previously, we can dispense with the scalar field in the limit $\xi \rightarrow \infty$. In this limit Eq. (130) reduces to the scalar curvaton expression in Eq. (128), as expected. However, if there is indeed a contribution to the curvature perturbation from a scalar field, this may affect the value of $f_{\mathrm{NL}}$ even if $\xi \gg 1$. The reason is easily understood from Eq. (129), which suggests that, at the end of scaling $\mathcal{P}_{\|,+} \ll \mathcal{P}_{\phi}$, since $M \rightarrow \hat{m} \gg H_{*}{ }^{5}$ Thus, if $\left(\hat{m} / 3 H_{*}\right)^{2}>\xi / 2 \gg 1$, we find from Eqs. (112) and (113)

$$
f_{\mathrm{NL}}=\xi^{2} \frac{5}{4 \hat{\Omega}_{A}}\left(\frac{\mathcal{P}_{+}}{\mathcal{P}_{\phi}}\right)^{2}=\frac{5}{4 \hat{\Omega}_{A}}\left[\frac{\xi}{2}\left(\frac{3 H_{*}}{\hat{m}}\right)^{2}\right]^{2}<\frac{5}{4 \hat{\Omega}_{A}},
$$

which is smaller than the scalar curvaton result.

\section{One-loop corrections}

In the above we considered only the tree level contribution to the spectrum and bispectrum of the vector field. In principle, one-loop corrections may also contribute significantly. Their contribution in the case of vector fields has been studied in Ref. [42], where it is shown that the one-loop corrections to spectrum and bispectrum dominate only if

$$
N_{A A}^{2} \mathcal{P}_{A}>N_{A}^{2},
$$

where $\mathcal{P}_{A} \equiv\left(2 \mathcal{P}_{+}+\mathcal{P}_{\|}\right)$and $N_{A A} \equiv\left|\boldsymbol{N}_{\boldsymbol{A} \boldsymbol{A}}\right|$, with

$$
N_{A A}^{i j} \equiv \frac{\partial^{2} N}{\partial W_{i} \partial W_{j}} .
$$

As shown in Ref. [22], for the vector curvaton we have

$$
N_{A}=\frac{2}{3} \frac{\hat{\Omega}_{A}}{W} \quad \text { and } \quad N_{A A}=2 \frac{\hat{\Omega}_{A}}{W^{2}} .
$$

Using the above we can recast Eq. (132) as

$$
\delta W \sim \sqrt{\mathcal{P}_{A}}>\frac{1}{3} W,
$$

which clearly violates our perturbative approach. Hence, we conclude that in the vector curvaton case the one-loop correction has to be subdominant.

\footnotetext{
5 This reflects the fact that the amplitude of the oscillating vector field perturbations becomes exponentially suppressed during inflation because it decreases as $a^{-3}$ before the end of scaling, cf. Eq. 821).
} 


\section{EVOLUTION OF THE ZERO MODE}

As is evident from Eq. (109), in order to calculate the curvature perturbation associated with the vector field one needs to study also the evolution of the homogeneous zero mode $W$. Combining Eqs. (2) and (9) and using Eq. (17), we obtain

$$
\ddot{\boldsymbol{W}}+3 H \dot{\boldsymbol{W}}+\left[\left(1-\frac{1}{2} \alpha\right) \dot{H}-\frac{1}{4}(\alpha+4)(\alpha-2) H^{2}+M^{2}\right] \boldsymbol{W}=0,
$$

where we also considered Eq. (3).

\section{A. During inflation}

As we have shown, to obtain a scale invariant spectrum for the transverse components of the vector field perturbations we require $f(a)$ to scale according to Eq. (19), i.e. $\alpha=-1 \pm 3$. Using this and considering de Sitter inflation (with $\dot{H} \approx 0$ ) the above becomes ${ }^{6}$

$$
\ddot{\boldsymbol{W}}+3 H \dot{\boldsymbol{W}}+M^{2} \boldsymbol{W}=0
$$

We show below that, when $M \ll H$ (true at early times when $\alpha=-4$; always true when $\alpha=2$ ), the solution of the above is well approximated by

$$
W \simeq \hat{C}_{1}+\hat{C}_{2} a^{-3}
$$

where $\hat{C}_{i}$ are constants. The dominant term to the solution of Eq. (138) is determined by the initial conditions. A natural choice of initial conditions for the vector field zero-mode can be based on energy equipartition grounds. As is demonstrated in what follows, if energy equipartition is assumed at the onset of inflation, the dominant term turns out to be the decaying mode, $W \propto a^{-3}$ when $\alpha=-4$ and the "growing" mode $W=$ constant, when $\alpha=2$.

To apply energy equipartition in the initial conditions we need to consider the energy-momentum tensor for this theory, which, from Eq. (1), is given by [11]

$$
T_{\mu \nu}=f\left(\frac{1}{4} g_{\mu \nu} F_{\rho \sigma} F^{\rho \sigma}-F_{\mu \rho} F_{\nu}{ }^{\rho}\right)+m^{2}\left(A_{\mu} A_{\nu}-\frac{1}{2} g_{\mu \nu} A_{\rho} A^{\rho}\right) .
$$

If we assume that the homogenised vector field lies along the $x^{3}$-direction, we can write the above as [11]

$$
T_{\mu}^{\nu}=\operatorname{diag}\left(\rho_{A},-p_{\perp},-p_{\perp},+p_{\perp}\right)
$$

where

$$
\rho_{A} \equiv \rho_{\text {kin }}+V_{A}, \quad p_{\perp} \equiv \rho_{\text {kin }}-V_{A}
$$

with

$$
\begin{aligned}
\rho_{\text {kin }} & \equiv-\frac{1}{4} f F_{\mu \nu} F^{\mu \nu}=\frac{1}{2} a^{-2} f \dot{A}^{2}=\frac{1}{2}\left[\dot{W}+\left(1-\frac{1}{2} \alpha\right) H W\right]^{2}, \\
V_{A} & \equiv-\frac{1}{2} m^{2} A_{\mu} A^{\mu}=\frac{1}{2} a^{-2} m^{2} A^{2}=\frac{1}{2} M^{2} W^{2},
\end{aligned}
$$

where $A \equiv|\boldsymbol{A}|$, we used Eqs. (9) and (17), and we assumed a negative signature for the metric.

Energy equipartition, therefore, corresponds to

$$
\left(\rho_{\mathrm{kin}}\right)_{0} \simeq\left(V_{A}\right)_{0}
$$

where the subscript ' 0 ' indicates the values at some initial time, e.g. near the onset of inflation.

\footnotetext{
${ }^{6}$ The equation of motion for the zero mode of the canonically normalised field is: $\ddot{\boldsymbol{B}}+H \dot{\boldsymbol{B}}+\left(-2 H^{2}+M^{2}\right) \boldsymbol{B}=0$, which agrees with the findings in Ref. [1].
} 


\section{Case: $f \propto a^{-4}$}

In this case $M \propto a^{3}$ and the solution to Eq. (137) is

$$
W=a^{-3}\left[\hat{C}_{3} \sin \left(\frac{M}{3 H}\right)+\hat{C}_{2} \cos \left(\frac{M}{3 H}\right)\right] .
$$

When $M \gtrsim H$ the above shows that the amplitude of the oscillating zero mode is decreasing as $\|W\| \propto a^{-3}$. In the opposite regime, when $M \ll H$ the solution above is well approximated by Eq. (138) with $\hat{C}_{1}=\hat{C}_{3} a_{0}^{-3} M_{0} / 3 H$, where we considered that $a^{-3} M=a_{0}^{-3} M_{0}=$ constant. Using this, the constants $\hat{C}_{2}$ and $\hat{C}_{3}$ in Eq. (145) can be expressed in terms of initial values of the field amplitude $W_{0}$ and its velocity $\dot{W}_{0}$ (in field space):

$$
\hat{C}_{2}=-\frac{\dot{W}_{0}}{3 H} a_{0}^{3} \quad \text { and } \quad \hat{C}_{3}=\frac{\left(\dot{W}_{0}+3 H W_{0}\right)}{M_{0}} a_{0}^{3} .
$$

Assuming initial equipartition of energy we can relate $W_{0}$ with $\dot{W}_{0}$. From Eqs. (142) and (143), setting $\alpha=-4$, we readily obtain

$$
\rho_{\text {kin }}=\frac{1}{2}(\dot{W}+3 H W)^{2} \quad \text { and } \quad V_{A}=\frac{1}{2} M^{2} W^{2} .
$$

Then, using Eq. (144), we get

$$
\dot{W}_{0} \simeq W_{0}\left(-3 H \pm M_{0}\right)
$$

Substituting this relation into Eq. (146) we find that the evolution of the vector field $W$ in Eq. (145) takes the simple form:

$$
W=W_{0}\left(\frac{a}{a_{0}}\right)^{-3} \sqrt{2} \cos \left(\frac{M}{3 H} \pm \frac{\pi}{4}\right) .
$$

Note that this equation is valid for any value of $M$. However, we can see that when $M \ll H$ the zero mode of the vector field is decreasing as $W \propto a^{-3}$, but when $M \gg H$ it oscillates rapidly with a decreasing amplitude proportional to $a^{-3}$. On this basis we can assume that the typical value of the zero mode during inflation always scales as

$$
W \propto a^{-3}
$$

With the assumption of initial equipartition of energy for the vector field at the onset of inflation we can calculate the kinetic and potential energy densities. ${ }^{7}$ Inserting Eq. (149) and its derivative into Eqs. (142) and (143) we find

$$
\rho_{\text {kin }}=\left[W_{0} M_{0} \sin \left(\frac{M}{3 H} \pm \frac{\pi}{4}\right)\right]^{2} \quad \text { and } \quad V_{\mathrm{A}}=\left[W_{0} M_{0} \cos \left(\frac{M}{3 H} \pm \frac{\pi}{4}\right)\right]^{2}
$$

Hence, the total energy density is constant

$$
\rho_{A}=M_{0}^{2} W_{0}^{2}
$$

Because this relation is independent of the vector field mass $M$ it is valid in both regimes: when $M \ll H$ and $W$ follows a power law evolution, and when $M \gg H$ and $W$ oscillates. This is valid as long as $f(a)$ and $m(a)$ are varying with time.

\footnotetext{
7 By "potential" we refer to the energy density stored in the mass-term $V_{A}=-\frac{1}{2} m^{2} A_{\mu} A^{\mu}$.
} 


\section{Case: $f \propto a^{2}$}

In this case, $M=$ constant, which means that the solution of Eq. (137) is

$$
W=a^{-3 / 2}\left[\hat{C}_{1} a^{\sqrt{\frac{9}{4}-\left(\frac{M}{H}\right)^{2}}}+\hat{C}_{2} a^{-\sqrt{\frac{9}{4}-\left(\frac{M}{H}\right)^{2}}}\right] .
$$

Since in this case $M \ll H$, the above solution is always well approximated by Eq. (138) and there is no oscillating regime.

Now, Eqs. (142) and (143) take the form

$$
\rho_{\text {kin }}=\frac{1}{2} \dot{W}^{2} \quad \text { and } \quad V_{A}=\frac{1}{2} M^{2} W^{2} .
$$

Combining Eqs. (138) and (154), we find

$$
\rho_{\text {kin }}=\frac{9}{2} H^{2} \hat{C}_{2}^{2} a^{-6}
$$

Thus, assuming energy equipartition at the onset of inflation (cf. Eq, (144)) gives

$$
\left(1+\frac{\hat{C}_{1}}{\hat{C}_{2}} a_{0}^{3}\right)^{2}=\left(\frac{3 H}{M_{0}}\right)^{2} \gg 1 \Rightarrow \hat{C}_{1} \simeq \pm \frac{3 H}{M_{0}} a_{0}^{-3} \hat{C}_{2},
$$

where we used that $M_{0}=M \ll H$. Inserting the above into Eq. (138) we find

$$
W=a_{0}^{-3} \hat{C}_{2}\left[\left(\frac{a_{0}}{a}\right)^{3} \pm \frac{3 H}{M_{0}}\right] \simeq \text { constant } \simeq W_{0},
$$

because, after the onset of inflation, $\left(a_{0} / a\right)^{3} \ll 1 \ll 3 H / M_{0}$.

Therefore, we have found that $W$ remains constant. Since $M=$ constant, this means that $V_{A}$ also remains constant. On the other hand, Eq. (155) suggests that $\rho_{\text {kin }} \propto a^{-6}$. Thus, since we assumed energy equipartition at the onset of inflation, we find that, during inflation, $\rho_{\text {kin }} \ll V_{A}$. Hence,

$$
\rho_{A} \approx V_{A} \simeq M_{0}^{2} W_{0}^{2}
$$

where $M=$ constant $=M_{0}$. This result is the same as in the case $f \propto a^{-4}$ in Eq. (152).

We should stress here that, according to the above, for $\alpha=-1 \pm 3$ the typical value of the zero mode scales as $W \propto a^{(\alpha / 2)-1}$, which means that the zero mode of the comoving vector field $A=a W / \sqrt{f}$ remains constant.

\section{B. After the end of scaling}

The simplest choice would be to assume that the scaling of $f(a)$ and $m(a)$ during inflation is terminated at the end of inflation. This would imply that $f$ and $m$ are modulated by a degree of freedom which varies during inflation, e.g. the inflaton field. We explore this possibility in Sec. IX Here we briefly comment on the possibility of allowing the scaling to end before the end of inflation.

At the end of scaling $f \rightarrow 1$ and $m \rightarrow \hat{m}=$ constant. Then, the zero-mode equation of motion can be obtained by setting $\alpha=0$ in Eq. (136). During de Sitter inflation this gives

$$
\ddot{\boldsymbol{W}}+3 H \dot{\boldsymbol{W}}+\left(2 H^{2}+\hat{m}^{2}\right) \boldsymbol{W}=0,
$$

which is solved by

$$
W=a^{-3 / 2}\left[\hat{C}_{1}^{\prime} a^{\sqrt{\frac{1}{4}-\left(\frac{\hat{m}}{H}\right)^{2}}}+\hat{C}_{2}^{\prime} a^{-\sqrt{\frac{1}{4}-\left(\frac{\hat{m}}{H}\right)^{2}}}\right]
$$

where $\hat{C}_{i}^{\prime}$ are constants. The end of scaling occurs before the onset of the oscillations if $\hat{m} \ll H$. In this case the above suggests that $W=\tilde{C}_{1}^{\prime} a^{-1}+\tilde{C}_{2}^{\prime} a^{-2}$, with $\tilde{C}_{i}^{\prime}$ constants. This is not of the same form with Eqs. (138) or (153). Therefore the transition at the end of scaling is not smooth but it is indeed felt by the zero mode if this occurs before the onset of the oscillations. 
The end of scaling occurs after the onset of the oscillations if $\hat{m} \gtrsim H$ (which is possible only in the case with $\alpha=-4$ ). In this case Eq. (160) suggests that the amplitude of the oscillations decreases as $\|W\| \propto a^{-3 / 2}$, which agrees with the findings of Ref. [1]. This is not in agreement with Eq. (145), which suggests that, during the oscillations, $\|W\| \propto a^{-3}$. Thus, even if the end of scaling takes place after the onset of the oscillations, the transition modifies the evolution of the zero-mode.

The vector field perturbations mimic the behaviour of the zero mode if the end of scaling occurs in the $r \gg 1$ regime, i.e. when $\hat{m} \gg k / a$. For such scales, after the end of scaling, the equations of motion for the transverse and longitudinal components, Eqs. (10) and (11), become identical and of the same form as Eq. (159). Thus, the mode functions satisfy the equation

$$
\ddot{w}_{\lambda}+3 H \dot{w}_{\lambda}+\left(2 H^{2}+\hat{m}^{2}\right) w_{\lambda}=0,
$$

which means that the typical value of the vector field perturbations is

$$
\delta W_{\lambda}=\sqrt{\mathcal{P}_{\lambda}} \propto w_{\lambda}=a^{-3 / 2}\left[\tilde{c}_{1} a^{\sqrt{\frac{1}{4}-\left(\frac{\hat{m}}{H}\right)^{2}}}+\tilde{c}_{2} a^{-\sqrt{\frac{1}{4}-\left(\frac{\hat{m}}{H}\right)^{2}}}\right],
$$

where $\tilde{c}_{i}$ are constants. Therefore, similarly to the above, the solution is not similar to Eqs. (55) and (71) for the transverse and longitudinal components respectively if $\hat{m} \ll H$. This is also true if $\hat{m} \gtrsim H$, i.e. when the end of scaling occurs after the onset of the oscillations, for which $\left\|\delta W_{\lambda}\right\| \propto a^{-3 / 2}$; in contrast to $\left\|\delta W_{\lambda}\right\| \propto a^{-3}$ before the end of scaling cf. Eq. (82). Note here that the spectrum for the scales that exit the horizon after the end of scaling is not scale invariant.

From the above we see that, if the end of the scaling occurs before the end of inflation the evolution of the zeromode and the vector field perturbations is non-trivially affected, which significantly complicates the treatment. Since attributing the scaling of $f(a)$ and $m(a)$ to some degree of freedom which varies during inflation but not afterward (e.g. the inflaton field, see Sec. IX] is much more physically motivated than the alternative considered above, in the following, we assume that this is indeed the case and the end of scaling occurs at the end of inflation.

\section{After inflation}

At the end of inflation we assume that the scaling of $f$ and $m$ has ended and we have

$$
f=1 \text { and } m=\hat{m} \text {. }
$$

Hence, Eqs. (152) and (158) no longer apply. The evolution of $\rho_{A}$ is determined as follows.

As mentioned already, after the end of scaling, $\alpha=0$ and $M=\hat{m}$. Then, Eqs. (142) and (143) become

$$
\rho_{\text {kin }}=\frac{1}{2}(\dot{W}+H W)^{2} \quad \text { and } \quad V_{A}=\frac{1}{2} \hat{m}^{2} W^{2} .
$$

The behaviour of $\rho_{\text {kin }}$ and $V_{A}$ depends on whether the vector field is light or not. To see this let us calculate the evolution of the field after inflation. With the conditions in Eq. (163) the physical vector field of Eq. (9) is $\boldsymbol{W}=\boldsymbol{A} / a$, while Eq. (136) becomes

$$
\ddot{\boldsymbol{W}}+3 H \dot{\boldsymbol{W}}+\left(\dot{H}+2 H^{2}+\hat{m}^{2}\right) \boldsymbol{W}=0
$$

where the Hubble parameter after inflation decreases as $H(t)=\frac{2}{3(1+w) t}$, with $w \equiv p / \rho$ being the barotropic parameter of the Universe. Solving Eq. (165) we find

$$
\begin{aligned}
W & =t^{\frac{1}{2}\left(\frac{w-1}{w+1}\right)}\left[\tilde{C}_{1} J_{d}(\hat{m} t)+\tilde{C}_{2} J_{-d}(\hat{m} t)\right] \quad \text { and } \\
\dot{W}+H W & =\hat{m} t^{\frac{1}{2}\left(\frac{w-1}{w+1}\right)}\left[\tilde{C}_{1} J_{d-1}(\hat{m} t)-\tilde{C}_{2} J_{1-d}(\hat{m} t)\right],
\end{aligned}
$$

where $d=\frac{1+3 w}{6(1+w)}$. One can easily see that the vector field behaves differently if it is light, $\hat{m} t \ll 1$, or heavy, $\hat{m} t \gg 1$.

Let us first see what happens if the vector field is light. Then, Eqs. (166) and (167) can be approximated as

$$
\begin{aligned}
W & =t^{\frac{1}{2}\left(\frac{w-1}{w+1}\right)}\left[\frac{\tilde{C}_{1}}{\Gamma(1+d)}\left(\frac{\hat{m} t}{2}\right)^{d}+\frac{\tilde{C}_{2}}{\Gamma(1-d)}\left(\frac{\hat{m} t}{2}\right)^{-d}\right] \quad \text { and } \\
\dot{W}+H W & =\hat{m} t^{\frac{1}{2}\left(\frac{w-1}{w+1}\right)}\left[d \frac{\tilde{C}_{1}}{\Gamma(1+d)}\left(\frac{\hat{m} t}{2}\right)^{d-1}-\frac{1}{1-d} \frac{\tilde{C}_{2}}{\Gamma(1-d)}\left(\frac{\hat{m} t}{2}\right)^{1-d}\right] .
\end{aligned}
$$


Although the solution has one decaying and one growing mode, it might happen that the decaying mode stays larger than the growing mode. To check this we calculate constants $\tilde{C}_{1}$ and $\tilde{C}_{2}$ by matching the above equations to the values $W_{\text {end }}$ and $\dot{W}_{\text {end }}$ at the end of inflation (denoted by 'end'). Thus, we find that

$$
\begin{aligned}
W & =\frac{2}{3 w+1}\left(\frac{a}{a_{\text {end }}}\right)^{\frac{1}{2(3 w-1)}}\left(W_{\text {end }}+\frac{\dot{W}_{\text {end }}}{H_{*}}\right) \text { and } \\
\dot{W}+H W & =H_{*}\left(\frac{a}{a_{\text {end }}}\right)^{-2}\left(W_{\text {end }}+\frac{\dot{W}_{\text {end }}}{H_{*}}\right),
\end{aligned}
$$

where $H_{*}$ is the inflationary Hubble scale. Plugging these solutions into Eq. (164) (and using that $\left.a^{3(1+w)} \propto t^{2}\right)$ we obtain

$$
\frac{V_{A}}{\rho_{\mathrm{kin}}}=\frac{4}{(3 w+1)^{2}}\left(\frac{\hat{m}}{H_{*}}\right)^{2}\left(\frac{t}{t_{\mathrm{end}}}\right)^{2} \simeq(\hat{m} t)^{2} \ll 1
$$

which implies that the total energy density of the light vector field is

$$
\rho_{A} \simeq \rho_{\text {kin }}=\frac{1}{2}\left(\dot{W}_{\text {end }}+W_{\text {end }} H_{*}\right)^{2}\left(\frac{a}{a_{\text {end }}}\right)^{-4} \Rightarrow \rho_{A} \propto a^{-4} .
$$

Therefore, we see that the energy density of the light vector field scales as that of relativistic particles. This is in striking difference to the scalar field case, in which when the field is light its density remains constant even after inflation.

On the other hand, if the vector field is heavy, $\hat{m} t \gg 1$, the Bessel functions in Eqs. (166) and (167) are oscillating and the latter can be recast as

$$
\begin{aligned}
W & =\sqrt{\frac{2}{\pi}} t^{-\frac{1}{1+w}}\left[\tilde{C}_{1} \cos \left(\hat{m} t-\frac{1+2 d}{4} \pi\right)+\tilde{C}_{2} \cos \left(\hat{m} t-\frac{1-2 d}{4} \pi\right)\right] \quad \text { and } \\
\dot{W}+H W & =\sqrt{\frac{2}{\pi}} \hat{m} t^{-\frac{1}{1+w}}\left[\tilde{C}_{1} \sin \left(\hat{m} t-\frac{1+2 d}{4} \pi\right)+\tilde{C}_{2} \sin \left(\hat{m} t-\frac{1-2 d}{4} \pi\right)\right] .
\end{aligned}
$$

As can be seen above, when the vector field is heavy, it oscillates with a very high frequency and with amplitude decreasing as $t^{-1 /(1+w)} \propto a^{-3 / 2}$. This was already demonstrated in Ref. [1]. When we calculate the energy density of the oscillating vector field from Eqs. (141) and (164) we find

$$
\rho_{A}=\frac{1}{\pi} \hat{m} t^{-\frac{2}{1+w}}\left[\tilde{C}_{1}^{2}+\tilde{C}_{2}^{2}+2 \tilde{C}_{1} \tilde{C}_{2} \cos (d \pi)\right] \Rightarrow \rho_{A} \propto a^{-3} .
$$

Thus, we see that the energy density of the heavy vector field scales as that of non-relativistic matter. Furthermore, if calculating the pressure from Eq. (141), we find that it oscillates with a high frequency:

$$
p_{\perp}=\frac{1}{\pi} \hat{m} t^{-\frac{2}{1+w}}\left[\tilde{C}_{1}^{2} \sin (2 \hat{m} t-d \pi)+\tilde{C}_{2}^{2} \sin (2 \hat{m} t+d \pi)+2 \tilde{C}_{1} \tilde{C}_{2} \sin (2 \hat{m} t)\right] \Rightarrow \overline{p_{\perp}} \approx 0 .
$$

Therefore, we have found that, on average, the oscillating vector field behaves as pressureless isotropic matter (see Eq. (140), which is in agreement with Ref. 1]. Hence, the massive vector field can dominate the Universe without generating excessive large scale anisotropy. This is crucial for the vector curvaton mechanism because, to produce the curvature perturbation, the field must dominate (or nearly dominate) the Universe without inducing anisotropic expansion.

\section{CURVATON PHYSICS}

In this section we calculate constraints for our vector curvaton model assuming that the scaling behaviour of $f(a)$ and $m(a)$ ends when inflation is terminated. This implies that the scaling is controlled by some degree of freedom which varies during inflation, e.g. the inflaton field. A specific example of this kind is discussed in Sec. IX 


\section{A. Basics}

According to the curvaton scenario 2] the total curvature perturbation can be calculated as the sum of individual curvature perturbations from the constituent components of the universe multiplied by the appropriate weighting factor. In our scenario this is written as follows

$$
\zeta=\left(1-\hat{\Omega}_{A}\right) \zeta_{\mathrm{rad}}+\hat{\Omega}_{A} \zeta_{A},
$$

where $\hat{\Omega}_{A}$ is defined in Eq. (110). As in the scalar curvaton paradigm, the above is to be evaluated at the time of decay of the curvaton field.

As was discussed in Sec. VI if $\hat{m} \gg H_{*}$ at the end of inflation, then the vector field perturbation spectrum is isotropic and may generate the total curvature perturbation in the Universe without violating observational bounds on the statistical anisotropy of the curvature perturbations. If this is the case, we can assume that $\zeta_{\text {rad }}=0$. On the other hand, when $\hat{m} \ll H_{*}$, the amplitude of the spectrum of the longitudinal component of the vector field perturbations is substantially larger than the one of the transverse perturbations. Hence, the curvature perturbation due to the vector field is excessively anisotropic. To avoid conflict with observational bounds (see Ref. 12]), the contribution of the vector field to the curvature perturbation has to remain subdominant. Therefore, for this scenario, we have to consider $\zeta_{\text {rad }} \neq 0$ and the curvature perturbation already present in the radiation dominated universe must dominate the one produced by the vector curvaton field.

For definiteness let us assume that inflation is driven by some inflaton field, which after inflation oscillates around its VEV until reheating, when it decays into relativistic particles. The vector field must be subdominant during this time. But after reheating, the Universe is radiation dominated with the energy density decreasing as $\rho_{\text {rad }} \propto a^{-4}$. If the vector field at this epoch is heavy and therefore undergoes rapid oscillations, its relative energy density increases, $\rho_{A} / \rho_{\text {rad }} \propto a$, as can be seen from Eq. (176). When the field becomes dominant (or nearly dominant) it can imprint its perturbation spectrum onto the Universe.

The contribution to the curvature perturbation by the vector field is calculated as follows. On the spatially flat slicing of spacetime we can write for each component of the content of the Universe

$$
\zeta_{n}=-H \frac{\delta \rho_{n}}{\dot{\rho}_{n}},
$$

where $n$ represents different components of the cosmic fluid. Using the continuity equation $\dot{\rho}_{n}=-3 H\left(\rho_{n}+p_{n}\right)$, one can recast the above equation for the vector field as

$$
\zeta_{A}=\left.\left.\left.\frac{\delta \rho_{A}}{3 \rho_{A}}\right|_{\mathrm{dec}} \approx \frac{2}{3} \frac{\|\delta W\|}{\|W\|}\right|_{\mathrm{dec}} \simeq \frac{2}{3} \frac{\delta W}{W}\right|_{\mathrm{end}},
$$

where we considered that the decay of the vector field (labelled by 'dec') occurs after inflation and after the onset of its oscillations so that it is pressureless, as shown in Eq. (177). In the last relation we took into account that, after inflation, the equations of motion are the same for the zero mode and for the superhorizon perturbations of the field.

In Sec. IVC it was shown that the typical value of the field perturbation is $\delta W \sim\left(3 H_{*} / M\right)\left(H_{*} / 2 \pi\right)$. If $M \ll H_{*}$ this is because the longitudinal component is dominant over the transverse ones (see Eq. (74)). If $M \gg H_{*}$, then the transverse and longitudinal components are oscillating with the same amplitudes (see Eq. (82)).

For this reason, at the end of inflation, we can write

$$
\delta W_{\mathrm{end}} \sim \frac{3 H_{*}}{\hat{m}} \frac{H_{*}}{2 \pi} \simeq \frac{H_{*}^{2}}{\hat{m}}
$$

where we have taken $M=\hat{m}$ and $f=1$ at the end of inflation. $W_{\text {end }}$ can be found from Eq. (152) by using $\left(\rho_{A}\right)_{\mathrm{end}} \simeq W_{0} M_{0} \simeq W_{\text {end }} \hat{m}$ (see Eqs. (152) and (158)). Thus,

$$
W_{\text {end }} \sim \frac{\sqrt{\left(\rho_{A}\right)_{\text {end }}}}{\hat{m}} .
$$

Hence, from Eq. (180) we calculate the curvature perturbation of the vector field

$$
\zeta_{A} \sim \Omega_{\mathrm{end}}^{-1 / 2} \frac{H_{*}}{m_{P}}
$$


where $\Omega_{\mathrm{end}} \equiv\left(\rho_{A} / \rho\right)_{\text {end }}$ is the density parameter of the vector field at the end of inflation, $\rho_{\mathrm{end}}$ is the total energy density dominated by the inflaton field, and we have used the Friedmann equation: $3 m_{P}^{2} H_{*}^{2}=\rho_{\text {end }}$. Since the vector field must be subdominant during inflation we have $\Omega_{\mathrm{end}} \ll 1$. Since we also require $\zeta_{A} \sim(\delta W / W)_{\text {end }}<1$ for our perturbative approach to be valid, we obtain the following range for $\Omega_{\mathrm{end}}$ :

$$
\left(\frac{H_{*}}{m_{P}}\right)^{2} \ll \Omega_{\mathrm{end}} \ll 1 .
$$

Eqs. (183) and (184) are valid in both $\alpha=-1 \pm 3$ cases. The only difference is that, in the $f \propto a^{2}$ case, statistically isotropic curvature perturbations cannot be generated. Hence, only the considerations for statistically anisotropic perturbations in Sec. VIIIB2 are relevant. $^{8}$

\section{B. The parameter space}

Here we calculate the parameter space for the model following the method of Ref. 11. First, we note that at the end of inflation the inflaton field starts oscillating and $w \neq-1$. Therefore the Hubble parameter decreases as $H(t) \sim t^{-1}$. In general, the inflaton potential is approximately quadratic around its VEV. Thus, the coherently oscillating inflaton field corresponds to a collection of massive particles (inflatons) whose energy density decreases as $a^{-3}$. When the Hubble parameter falls bellow the inflaton decay rate $\Gamma$, the inflaton particles decay into much lighter relativistic particles reheating the Universe. After reheating, the Universe becomes radiation dominated with energy density scaling as $\rho_{\text {rad }} \propto a^{-4}$.

On the other hand, the evolution of the energy density of the vector field, depends on its mass $\hat{m}$. As discussed in Sec. VII] if $\hat{m} \ll H_{*}$ the energy density scales as $\rho_{A} \propto a^{-4}$ until the vector field becomes heavy and starts oscillating. If $\hat{m} \gg H_{*}$, however, the vector field has already started oscillating during inflation and $\rho_{A} \propto a^{-3}$.

To avoid causing an excessive anisotropic expansion period the vector field must be oscillating before it dominates the Universe and decays. This requirement implies that

$$
\Gamma, \hat{m}>\Gamma_{A}, H_{\text {dom }},
$$

where $\Gamma_{A}$ is the decay rate of the vector field and $H_{\mathrm{dom}}$ is the value of the Hubble parameter when the vector field dominates the Universe if it has not decayed already. Working as in Ref. [11], we can estimate $H_{\text {dom }}$ as

$$
H_{\mathrm{dom}} \sim \Omega_{\mathrm{end}} \Gamma^{1 / 2} \min \left\{1 ; \frac{\hat{m}}{H_{*}}\right\}^{2 / 3} \min \left\{1 ; \frac{\hat{m}}{\Gamma}\right\}^{-1 / 6} .
$$

Similarly, if the vector field decays before it dominates, the density parameter just before the decay is given by

$$
\Omega_{\mathrm{dec}} \sim \Omega_{\mathrm{end}}\left(\frac{\Gamma}{\Gamma_{A}}\right)^{1 / 2} \min \left\{1 ; \frac{\hat{m}}{H_{*}}\right\}^{2 / 3} \min \left\{1 ; \frac{\hat{m}}{\Gamma}\right\}^{-1 / 6} .
$$

where $\Omega_{\mathrm{dec}} \equiv\left(\Omega_{A}\right)_{\mathrm{dec}}$. Combining the last two equations and using Eq. (183) we can express the inflationary Hubble scale as

$$
\frac{H_{*}}{m_{P}} \sim \Omega_{\mathrm{dec}}^{1 / 2} \zeta_{A} \min \left\{1 ; \frac{\hat{m}}{H_{*}}\right\}^{-1 / 3} \min \left\{1 ; \frac{\hat{m}}{\Gamma}\right\}^{1 / 12}\left(\frac{\max \left\{\Gamma_{A} ; H_{\mathrm{dom}}\right\}}{\Gamma}\right)^{1 / 4}
$$

The bound on the inflationary scale can be obtained by considering that the decay rate of the vector field is $\Gamma_{A} \sim h^{2} \hat{m}$, where $h$ is the coupling to the decay products. Then we can write $\max \left\{\Gamma_{A} ; H_{\mathrm{dom}}\right\} \gtrsim h^{2} \hat{m}$. Furthermore, we must consider the possibility of thermal evaporation of the vector field condensate during the radiation dominated phase. If this were to occur, all the memory of the superhorizon perturbation spectrum would be erased. The thermalisation rate is determined by the scattering rate of the massive boson field with the thermal bath and is given

\footnotetext{
8 The lower bound in Eq. (184) guarantees that $\delta W / W \ll 1$ throughout inflation. The reason is the following. As discussed before Eq. (181), during inflation $\delta W / W \sim H_{*}^{2} / M W$. Now, for $f \propto a^{-1 \pm 3}$ and $m \propto a$ we have $M=m / \sqrt{f} \propto a^{-\frac{3}{2}(-1 \pm 1)}$. Also, from Eqs. (150) and 157] we obtain $W \propto a^{\frac{3}{2}(-1 \pm 1)}$. Thus, we see that, in all cases considered $M W=$ constant, which means that $\delta W / W=$ constant during inflation. Therefore, $(\delta W / W)_{\text {end }} \ll 1$ is sufficient to guarantee the validity of our perturbative approach throughout inflation.
} 
by $\Gamma_{\mathrm{sc}} \sim h^{4} T$. Requiring that the condensate does not thermalise before it decays, $\Gamma_{\mathrm{sc}}<\Gamma_{A}$, in Ref. [10] it was shown that $h$ must satisfy

$$
\frac{\hat{m}}{m_{P}} \lesssim h \lesssim\left(\frac{\hat{m}}{m_{P}}\right)^{1 / 4}
$$

The lower bound in the above is due to decay through gravitational interactions, while the upper bound becomes irrelevant if the vector field dominates the Universe before it decays (then $h \lesssim 1$ is sufficient). This is because, in the latter case, the energy density of the thermal bath is exponentially smaller than $\rho_{A}$ and the vector field condensate does not evaporate.

From Eq. (188) one can see that the parameter space is maximised if the Universe undergoes prompt reheating after inflation, i.e. if $\Gamma \rightarrow H_{*}$. To find the parameter space we investigate two separate cases: when $\hat{m} \gg H_{*}$ and when $\hat{m} \ll H_{*}$.

\section{Statistically isotropic perturbations}

This possibility can be realised only in the case when $\alpha=-4$. As mentioned before, if the mass of the vector field at the end of inflation is larger than the Hubble parameter, $\hat{m}>H_{*}$, then the field has started oscillating already during inflation. In this case the amplitudes of the longitudinal and transverse perturbations are equal and therefore the curvature perturbations induced by the vector field are statistically isotropic. We can assume, in this case, that the vector field alone is responsible for the total curvature perturbation in the Universe without the need to invoke additional perturbations from other fields. Thus, we can set $\zeta_{\mathrm{rad}}=0$ in Eq. (178) and write

$$
\zeta \sim \Omega_{\mathrm{dec}} \zeta_{A} .
$$

Using this and the lower bound on $h$ we find from Eq. (189) the lower bound for the inflationary Hubble parameter

$$
\frac{H_{*}}{m_{P}} \gtrsim\left(\frac{\zeta}{\sqrt{\Omega_{\mathrm{dec}}}}\right)^{4 / 5}\left(\frac{\hat{m}}{m_{P}}\right)^{3 / 5}
$$

where we have taken into account that the parameter space is maximised when the universe undergoes prompt reheating, i.e. $\Gamma \rightarrow H_{*}$. From this expression it is clear that the lowest bound is attained when the vector field dominates the Universe before its decay, $\Omega_{\mathrm{dec}} \rightarrow 1$, and when the oscillations of the vector field commence at the very end of inflation, i.e. $\hat{m} \rightarrow H_{*}$. With these values we find the bounds

$$
H_{*} \gtrsim 10^{9} \mathrm{GeV} \quad \Leftrightarrow \quad V_{*}^{1 / 4} \gtrsim 10^{14} \mathrm{GeV}
$$

where $V_{*}^{1 / 4}$ denotes the inflationary energy scale and we used that $\zeta \approx 5 \times 10^{-5}$ from the observations of the Cosmic Background Explorer.

In view of the above, we can obtain a lower bound for the decay rate of the vector field. Indeed, using Eqs. (189) and (192) we find

$$
\Gamma_{A} \gtrsim \frac{\hat{m}^{3}}{m_{P}^{2}} \gtrsim \frac{H_{*}^{3}}{m_{P}^{2}} \gtrsim 10^{-9} \mathrm{GeV}
$$

From the above we find that the temperature of the Universe after the decay of the vector field is $T_{\mathrm{dec}} \sim \sqrt{m_{P} \Gamma_{A}} \gtrsim 10^{4} \mathrm{GeV}$, which is comfortably higher than the temperature at Big Bang Nucleosynthesis (BBN) $T_{\mathrm{BBN}} \sim 1 \mathrm{MeV}$ (i.e. the decay occurs much earlier than $\mathrm{BBN}$ ), and also higher than the electroweak phase transition, i.e. the decay precedes possible electroweak baryogenesis processes.

Since $\hat{m}>H_{*}$, Eq. (192) corresponds to a lower bound on $\hat{m}$. An upper bound on $\hat{m}$ can be obtained as follows. Because, $\hat{m}>H_{*} \gtrsim \Gamma$, Eq. (187) becomes

$$
\Omega_{\mathrm{dec}} \sim \Omega_{\mathrm{end}} \sqrt{\frac{\Gamma}{\Gamma_{A}}} .
$$

From Eq. (189) we have $\Gamma_{A} \gtrsim \hat{m}^{3} / m_{P}^{2}$. Combining this with the above we obtain

$$
\hat{m}^{3} \lesssim\left(\frac{\Omega_{\mathrm{end}}}{\Omega_{\mathrm{dec}}}\right) \Gamma m_{P}^{2}
$$


Now, when $\alpha=-4$ we have $M \propto a^{3}$ during inflation. Since the end of scaling occurs when inflation is terminated, for $a<a_{\text {end }}$ we can write

$$
\hat{m}=\left(\frac{a_{\mathrm{end}}}{a}\right)^{3} M \simeq e^{3 N_{\mathrm{osc}}} H_{*}
$$

where we considered that the field begins oscillating when $M \simeq H_{*}$ and $N_{\text {osc }}$ is the number of remaining e-folds of inflation when the oscillations begin. Inserting the above into Eq. (195) we find

$$
N_{\mathrm{osc}} \lesssim N_{\mathrm{osc}}^{\max } \equiv \frac{2}{9}\left[\ln \left(\frac{\Omega_{\mathrm{end}}}{\Omega_{\mathrm{dec}}}\right)+\ln \sqrt{\frac{\Gamma}{H_{*}}}+\ln \left(\frac{m_{P}}{H_{*}}\right)\right]<\frac{2}{9} \ln \left(\frac{m_{P}}{\Omega_{\mathrm{dec}} H_{*}}\right)
$$

where, in the last inequality, we used that $\Omega_{\mathrm{end}}<1$ and $\Gamma \lesssim H_{*}$. Now, considering that $\hat{m} \gtrsim H_{*}$, Eq. (191) gives

$$
\frac{\Omega_{\mathrm{dec}} H_{*}}{m_{P}} \gtrsim \zeta^{2}
$$

Hence, combining Eqs. (197) and (198) we obtain

$$
N_{\mathrm{osc}}^{\max }<-\frac{4}{9} \ln \zeta=4.4
$$

Thus, in view of Eq. (196), we obtain the bound $\hat{m} \lesssim e^{3 N_{\mathrm{osc}}^{\max }} H_{*}$, which results in the following parameter space for m:

$$
1 \lesssim \hat{m} / H_{*}<10^{6}
$$

where we used Eq. (199). The above range is reduced if the decay of the curvaton occurs more efficiently than through gravitational couplings, i.e. if $h>\hat{m} / m_{P}$. Nevertheless, we see that the parameter space in which the vector field undergoes isotropic particle production and can alone account for the curvature perturbation, is not small but may well be exponentially large. Indeed, repeating the above calculation with $\Gamma_{A} \sim \hat{m}$ (i.e. $h \sim 1$ ) it is easy to find that

$$
N_{\mathrm{osc}}=\frac{2}{3}\left[\ln \left(\frac{\Omega_{\mathrm{end}}}{\Omega_{\mathrm{dec}}}\right)+\ln \sqrt{\frac{\Gamma}{H_{*}}}\right] .
$$

Hence, using that $\Omega_{\text {end }}<1$ and $\Gamma \lesssim H_{*}$ we obtain

$$
N_{\mathrm{osc}}^{\max }=-\frac{2}{3} \ln \Omega_{\mathrm{dec}} \lesssim 3.1 \Rightarrow 1 \lesssim \hat{m} / H_{*}<10^{4}
$$

where we used $\Omega_{\mathrm{dec}} \gtrsim 10^{-2}$. This is because, in the case considered, $f_{\mathrm{NL}}$ is given by Eq. (128), so a smaller $\Omega_{\mathrm{dec}}$ would violate the current observational bounds on the non-Gaussianity in the CMB temperature perturbations [41].

Still, it seems that, to obtain an exponentially large parameter space for $\hat{m}$, we need $\rho_{A}$ not to be too much smaller than $V_{*}$ during inflation and also inflationary reheating to be efficient. In the case of gravitational decay $\left(\Gamma_{A} \sim \hat{m}^{3} / m_{P}^{2}\right)$ Eq. (197) has a weak dependence on both $\Omega_{\text {end }}$ and $\Gamma: \hat{m} \propto\left(\Omega_{\text {end }}^{2} \Gamma\right)^{1 / 3}$, which means that the allowed range of values for $\hat{m}$ remains large even when $\Omega_{\text {end }}$ and $\Gamma$ are substantially reduced. This is not necessarily so when $\Gamma_{A} \sim h^{2} \hat{m}$, with $h \gg \hat{m} / m_{P}$. Indeed, in this case it can be easily shown that $\hat{m} \propto h^{-2} \Omega_{\text {end }}^{2} \Gamma$. Therefore, if $\Gamma$ is very small it may eliminate the available range for $\hat{m}$. Fortunately, the decay coupling $h$ can counteract this effect without being too small.

\section{Statistically anisotropic perturbations}

If the vector field is not responsible for the total curvature perturbation in the Universe, the parameter space is more relaxed. In this case, the vector field may start oscillating after inflation and hence its mass is $\hat{m} \ll H_{*}$. However, this means that the curvature perturbation due to the vector field is strongly statistically anisotropic. For this reason we can no longer set $\zeta_{\text {rad }}$ to zero because the curvature perturbation present in the radiation dominated Universe must be dominant. In other words, the parameter $\xi$ defined in Eq. (106) needs to be very small, $\xi \ll 1$. 
From the Eq. (74) it is clear that if inflation ends when $M=\hat{m} \ll H_{*}$, the longitudinal power spectrum is much greater than the transverse ones, $\mathcal{P}_{\|} \gg \mathcal{P}_{+}$. Thus, from Eq. (104) we find

$$
g \simeq \frac{N_{A}^{2} \mathcal{P}_{\|}}{\mathcal{P}_{\zeta}^{\text {iso }}} \simeq \xi \frac{\mathcal{P}_{\|}}{\mathcal{P}_{\phi}}
$$

where we also considered Eq. (105) with $\xi \ll 1$. Since the anisotropic contribution to the spectrum is subdominant [12], to first order we can write

$$
\frac{\zeta^{2}}{\delta W_{\|}^{2}} \simeq \frac{\mathcal{P}_{\zeta}^{\text {iso }}}{\mathcal{P}_{\|}}
$$

Hence,

$$
N_{A}^{2} \delta W_{\|}^{2} \simeq g \zeta^{2}
$$

Now, from the $\delta N$ formalism at tree level we have [22] ${ }^{9}$

$$
\zeta=N_{\phi} \delta \phi+N_{A} \delta W
$$

Comparing the above with Eq. (178) we can equate the contributions to $\zeta$ from the vector field. Thus, we find

$$
N_{A} \delta W=\hat{\Omega}_{A} \zeta_{A}
$$

Using that

$$
\frac{\delta W^{2}}{\delta W_{\|}^{2}}=\frac{\sum_{\lambda} \delta W_{\lambda}^{2}}{\delta W_{\|}^{2}} \simeq \frac{2 \mathcal{P}_{+}+\mathcal{P}_{\|}}{\mathcal{P}_{\|}} \approx 1
$$

and combining Eqs. (205) and (207) we obtain

$$
\zeta \sim g^{-1 / 2} \Omega_{A} \zeta_{A}
$$

Inserting this expression into Eq. (188) and considering again that the lowest decay rate of the vector field is through gravitational decay, $\max \left\{\Gamma_{A} ; H_{\text {dom }}\right\} \geq \hat{m}^{3} / m_{P}^{2}$ we find

$$
\frac{H_{*}}{m_{P}}>\left(\frac{g \zeta^{2}}{\Omega_{\mathrm{dec}}}\right)^{3 / 4}\left(\frac{\hat{m}}{m_{P}}\right)^{5 / 8}\left(\frac{\Gamma}{m_{P}}\right)^{-3 / 8} \min \left\{1 ; \frac{\hat{m}}{\Gamma}\right\}^{1 / 8}
$$

The above suggests that the lower bound on $H_{*}$ is minimised for prompt reheating with $\Gamma \rightarrow H_{*}$. Also, from observations we know that the statistically anisotropic contribution to the curvature perturbation must be subdominant. Thus, the vector field should not dominate the Universe before its decay. Hence, using $\Gamma \rightarrow H_{*}$ and $\Omega_{A}<1$ we obtain

$$
\frac{H_{*}}{m_{P}}>\sqrt{g} \zeta \sqrt{\frac{\hat{m}}{m_{P}}} .
$$

From this expression it is clear that the parameter space for $H_{*}$ is maximised for the lowest mass value. The minimum mass of the vector field can be estimated from the requirement that the field decays before BBN. Because the lowest decay rate is the gravitational decay, this condition reads $\hat{m}^{3} / m_{P}^{2} \gtrsim T_{\mathrm{BBN}}^{2} / m_{P}$, with $T_{\mathrm{BBN}} \sim 1 \mathrm{MeV}$, which corresponds to $\hat{m} \gtrsim 10^{4} \mathrm{GeV}$. Using this, we find that the parameter space for the vector curvaton model with the statistically anisotropic curvature perturbations is

$$
H_{*}>\sqrt{g} 10^{7} \mathrm{GeV} \quad \Leftrightarrow \quad V_{*}^{1 / 4}>g^{1 / 4} 10^{13} \mathrm{GeV},
$$

\footnotetext{
${ }^{9}$ Technically, here we should have $N_{A} \delta W \rightarrow \boldsymbol{N}_{\boldsymbol{A}} \cdot \delta \boldsymbol{W}$ but the dot-product is dominated by one term, that of the longitudinal component, since $\delta W \simeq \delta W_{\|}$as shown in Eq. 208.
} 
i.e. it is somewhat relaxed compared to the statistically isotropic case (cf. Eq. (192)) depending on the magnitude of the statistical anisotropy in the spectrum, for which $g \lesssim 0.3$ [12]. This result is valid for both $\alpha=-1 \pm 3$ cases. From the above it is evident that there is ample parameter space for the mass of the vector field

$$
10 \mathrm{TeV} \lesssim \hat{m} \ll H_{*}
$$

We can readily use the above to briefly discuss an indicative example. Suppose that $\hat{m} \sim 10^{-2} H_{*}$. Then, from Eq. (211) we obtain $H_{*}>10^{-2} g \zeta^{2} m_{P}$. Assume now that statistical anisotropy in the power spectrum is observed with $g \sim 0.01$. Thus, we obtain $H_{*}>10^{-13} m_{P} \sim 100 \mathrm{TeV}$. Let us assume also that non-Gaussianity is observed with predominant angular modulation that peaks in the equilateral configuration with amplitude $\left\|f_{\mathrm{NL}}^{\text {equil }}\right\| \sim 10$. Then, according to Eq. (125), we have $g^{2} / \Omega_{\mathrm{dec}} \sim 10$, i.e. $\Omega_{\mathrm{dec}} \sim 10^{-5}$. Also, it is easy to check that $\Omega_{\mathrm{end}} \sim \Omega_{\mathrm{dec}} \sqrt{\Gamma_{A} / \hat{m}}$ comfortably satisfies the bound in Eq. (184) if $\Gamma_{A}>T_{\mathrm{BBN}}^{2} / m_{P}$. Finally, from the above we can estimate the amplitude of the non-Gaussianity in the local configuration $\left\|f_{\mathrm{NL}}^{\text {local }}\right\| \sim 10^{-3}$ [cf. Eq. (125)] and also $f_{\mathrm{NL}}^{\text {iso }} \sim 10^{-7}$ [cf. Eq. (124)].

\section{Constraints from isocurvature perturbations}

As discussed, when $\hat{m} \ll H_{*}$, particle production is strongly anisotropic and the curvaton has to be subdominant when it decays. The fact that the curvaton decays while subdominant allows the possibility to generate a sizable isocurvature perturbation, which needs to comply with observational constraints. Of course, if the curvaton decays early enough into relativistic particles which join the preexisting thermal bath then there is a possibility that no isocurvature perturbation is generated. For this to be so we require all the components of the late time Universe to be relativistic and in thermal equilibrium so that they can be produced by the decay products of the vector curvaton field. Hence, dark matter needs to be thermal. For WIMP dark matter we require curvaton decay to occur before the breaking of electroweak unification (i.e. the temperature at curvaton decay should be $T_{\text {dec }}>1$ TeV) so that the decay products can produce WIMPs. In this case baryons are also generated by the curvaton decay products and so are neutrinos. However, if the vector curvaton decay occurs later or if the dark matter is not thermal (e.g. axions) then an isocurvature perturbation can be generated. An estimate of the maximum isocurvature perturbation is then given by

$$
\mathcal{S}_{\max }=\left.\frac{\delta \rho}{\rho}\right|_{\text {after }}-\left.\frac{\delta \rho}{\rho}\right|_{\text {before }} \approx \frac{2}{5}\left[\left(\zeta_{\text {bef }}+\Omega_{\mathrm{dec}} \zeta_{A}\right)-\zeta_{\text {bef }}\right]=\frac{2}{5} \Omega_{\mathrm{dec}} \zeta_{A},
$$

where $\Omega_{\text {dec }} \ll 1, \quad(\delta \rho / \rho)_{\text {before }} \approx \frac{2}{5} \zeta_{\text {bef }}$ corresponds to the perturbation which preexists curvaton decay, while $(\delta \rho / \rho)_{\text {after }} \approx \frac{2}{5}\left(\zeta_{\text {bef }}+\Omega_{\text {dec }} \zeta_{A}\right)$ [c.f Eq. (178)] corresponds to the perturbation after curvaton decay which includes the contribution to the perturbation due to the curvaton. The above assumes that one of the constituents of the Universe content (e.g. dark matter) does not receive any contribution from the curvaton decay products while another such constituent receives $100 \%$ contribution, hence this is the maximum isocurvature perturbation (Realistically, $\mathcal{S}$ is model dependent and it is determined by the branching ratio of the vector curvaton decay to the various constituents of the Universe content).

Observational constraints require that an uncorrelated isocurvature perturbation cannot exceed $11 \%$ of the adiabatic mode [43]. This means that

$$
\Omega_{\mathrm{dec}} \zeta_{A} \lesssim 0.1 \zeta \Rightarrow g \lesssim 0.01
$$

where we considered also Eq. (209). Thus, even the maximum possible isocurvature perturbation produced by our model allows the generation of statistical anisotropy at the level of a few percent.

It is important to note here that the isocurvature perturbation is uncorrelated to the adiabatic one in contrast to the scalar curvaton model. This is because, in our model, when $\hat{m} \ll H_{*}, \zeta$ is not generated by the vector curvaton field but it preexists curvaton decay. The vector curvaton contribution to $\zeta$ is negligible (i.e. $\zeta \approx \zeta_{\text {bef }}$ ) and its effect amounts to generating statistical anisotropy only. In the scalar curvaton model, however, it is the curvaton field that generates $\zeta$ (i.e. $\zeta_{\text {bef }}$ is negligible), which means that, when curvaton decay occurs before domination, the isocurvature perturbation is fully (anti)correlated with the one of the curvaton.

\section{SCALAR FIELDS AS MODULATORS}

Throughout this paper we have taken the modulation of the kinetic function $f$ and the mass of the vector field for granted and assumed that it is due to some degree of freedom which varies during inflation. The most natural choice 
for such a degree of freedom is, of course, the inflaton field itself but other choices are also possible. In this section we briefly explore a couple of such possibilities, inspired by beyond the standard model theories such as supergravity or superstrings.

\section{A. The inflaton as modulator}

In string theory the modulation of parameters such as masses or kinetic functions is due to so-called moduli fields. The moduli are scalar fields which parametrise the size and shape of the extra dimensions. In that sense they are not fundamental scalar fields, but appear so from the view-point of the 4-dimensional observer. Typically, the dependence of masses and couplings on canonically normalised (i.e. with canonical kinetic terms) moduli fields is exponential.

Consider the following kinetic function

$$
f(\phi) \propto e^{-\frac{\alpha}{\mu} \phi / m_{P}},
$$

where $\mu>0$ is a real constant. Comparing this with Eq. (17) we see that

$$
m \propto a \propto e^{-\frac{1}{\mu} \phi / m_{P}},
$$

where we considered also Eq. (40). From the above we readily obtain

$$
\dot{\phi}=-\mu H m_{P}
$$

which suggests that

$$
\left(\rho_{\phi}\right)_{\mathrm{kin}} \equiv \frac{1}{2} \dot{\phi}^{2}=\frac{1}{6} \mu^{2} \rho
$$

and we have used the flat Friedmann equation $\rho=3\left(H m_{P}\right)^{2}$.

Now, suppose that $\phi$ is also driving quasi-de Sitter inflation, with $H \simeq$ constant. This requires $\rho=\rho_{\phi} \simeq V(\phi) \gg\left(\rho_{\phi}\right)_{\text {kin }}$, which demands

$$
\mu<\sqrt{6}
$$

To drive quasi-de Sitter inflation a scalar field needs to follow a slow-roll attractor solution, in which the acceleration term $\ddot{\phi}$ in its Klein-Gordon equation of motion is negligible. Thus, the field equation for the inflaton is the well known slow-roll equation

$$
3 H \dot{\phi} \simeq-V^{\prime}(\phi)
$$

where the prime denotes derivative with respect to $\phi .10$ Combining the above with Eq. (218) and taking $V(\phi) \simeq 3\left(H m_{P}\right)^{2}$ we obtain

$$
V(\phi) \simeq V_{0} e^{\mu \phi / m_{P}} \propto a^{-\mu^{2}}
$$

where $V_{0}$ is a density scale and we used Eq. (217). Thus, we find that the inflaton is characterised by an exponential potential, which is reasonable for a modulus field. The above also suggests that, for quasi-de Sitter inflation, when $V(\phi) \simeq \rho_{\phi}=\rho \simeq$ constant, we need to have

$$
\mu<1
$$

10 Because $\rho_{A} \ll V_{*}$ we can ignore the backreaction of the vector field to the scalar field dynamics. Indeed, if the modulation of $f$ and $m$ are due to a single scalar field $\phi$ then the Klein-Gordon equation of motion of the latter obtains a source term of the form

$$
\frac{\partial \mathcal{L}}{\partial \phi}=-\frac{1}{4} f^{\prime} F_{\mu \nu} F^{\mu \nu}+m m^{\prime} A_{\mu} A^{\mu}=\frac{H}{\dot{\phi}}\left(\alpha \rho_{\mathrm{kin}}-2 V_{A}\right) \sim-\frac{H}{\dot{\phi}} \rho_{A},
$$

where $\mathcal{L}$ is given in Eq. (1) and we considered $f \propto m^{\alpha}$ and $m \propto a$. If $\rho_{A}$ is small enough then the above can be negligible compared with the $-V^{\prime}$ source term in the equation of motion. 
which is somewhat stronger than the bound in Eq. (220). Combining Eqs. (221) and (222) we get

$$
\Delta \phi=\frac{2}{\mu} m_{P} \ln \left(1-\frac{1}{2} \mu^{2} \Delta N\right),
$$

where $\Delta N=H \Delta t$ is the number of the elapsing e-folds during which the inflaton varies by $\Delta \phi$. Notice that the above is consistent with Eq. (218) but it is valid only when $\mu^{2} \ll 2 / \Delta N$. ${ }^{11}$ The violation of this condition is equivalent with the violation of the "robustness" assumption for $H$ (i.e $H \simeq$ constant). That is, it corresponds to a significant variation in $H$, i.e. $-\Delta H \sim H^{2} \Delta t$. Technically, this can occur even under slow-roll conditions if $\Delta t$ is large enough. Since a "robust" $H$ is necessary for the generation of a scale invariant spectrum of perturbations, it should last at least as much as it takes for the entire range of the observable cosmological scales to exit the horizon. This range corresponds to about $\Delta N \simeq 9$ e-folds, which induces the bound

$$
\mu<\frac{\sqrt{2}}{3},
$$

which is tighter than the bounds in Eqs. (220) and (223).

Let us attempt to obtain an estimate of $\mu$. The slow roll parameters for the model in Eq. (222) are

$$
\begin{aligned}
\varepsilon & \equiv-\dot{H} / H^{2} \simeq \frac{1}{2} m_{P}^{2}\left(\frac{V^{\prime}}{V}\right)^{2}=\frac{1}{2} \mu^{2}, \\
\eta_{\phi} & \equiv m_{P}^{2} \frac{V^{\prime \prime}}{V}=\mu^{2} .
\end{aligned}
$$

If the inflaton is the source of the dominant contribution to the curvature perturbation of the Universe then, the spectral index of $\mathcal{P}_{\zeta}$ is

$$
n_{s}-1=2 \eta_{\phi}-6 \varepsilon=-\mu^{2}=-2 \varepsilon
$$

If, on the other hand, the dominant contribution to the curvature perturbation of the Universe is due to the vector curvaton (only possible in the $\alpha=-4$ case with $\hat{m} \gtrsim H_{*}$ ) then we have

$$
n_{s}-1=2 \eta_{A}-2 \varepsilon \approx-2 \varepsilon,
$$

where we considered that $\eta_{A}$ is negligible. Indeed, in almost all cases studied in the previous sections we had $\eta_{A}=0$ when $\epsilon_{f}, \epsilon_{m}=0$, the reason being that the power spectra for the vector field perturbations are exactly scale invariant, when inflation is considered to be de Sitter (i.e. $H=$ constant), which means that any deviations from scale invariance would be due to $\varepsilon \neq 1$ only. The only exception was the transverse component in the case when $\alpha=2$, where the $\eta_{A}$ parameter (defined in Eq. (91)) may not be negligible. However, in the following, we assume that $M \ll H$ is sufficiently small to ignore $\eta_{A}$ even in this case.

From Eqs. (228) and (229) we see that, regardless of whether the dominant contribution to the curvature perturbation is from the inflaton or from the vector curvaton field, the spectral index is

$$
n_{s}=1-2 \varepsilon .
$$

Comparing the above with the observed value [41] $n_{s}=0.960 \pm 0.014$ (for negligible tensors) we find

$$
\mu=0.200 \pm 0.036
$$

which satisfies the bound in Eq. (225). In view of Eq. (224), the above value guarantees that $H$ remains "robust" for about $\Delta N \simeq 50$, which comfortably encompasses the cosmological scales.

Note that, for the model in Eq. (222), the slow-roll parameters in Eqs. (226) and (227) remain constant and smaller than unity. Hence, inflation never ends. One can remedy this by either invoking some kind of hybrid mechanism to remove the inflaton from the slow-roll trajectory and send it to the true vacuum or by modifying the model such that slow-roll naturally breaks down eventually. ${ }^{12}$

\footnotetext{
${ }^{11}$ It turns out that Eq. 218 remains always valid even if Eq. 224) is not.

12 For example, in the toy-model with inflaton scalar potential $V(\phi)=V_{0}\left[\cosh \left(\mu \phi / m_{P}\right)-1\right]$ the slow-roll parameters are $\eta=\frac{1}{2} \mu^{2}+\varepsilon$ and $\varepsilon=\frac{1}{2} \mu^{2} \operatorname{coth}^{2}\left(\mu \phi / 2 m_{P}\right)$. The model approximates Eq. 222 when $\phi \gg m_{P} / \mu$ but allows inflation to end at $\phi_{\text {end }}=\frac{1}{\mu} m_{P} \ln \left(\frac{\sqrt{2}+\mu}{\sqrt{2}-\mu}\right) \simeq \sqrt{2} m_{P}$, where $\varepsilon\left(\phi_{\text {end }}\right) \equiv 1$ and we considered $\mu \ll 1$ in the last equality.
} 


\section{B. Higgsed vector curvaton}

Another possibility is that the vector field is Higgsed, which means that $m \propto \varphi$, where $\varphi$ is a Higgs field. Hence, from Eq. (40) we require $\varphi \propto a$ during inflation. Suppose that $\varphi$ is rolling down a hilltop potential of the form

$$
V(\varphi)=V_{\mathrm{top}}-\frac{1}{2} m_{\varphi}^{2} \varphi^{2}+\cdots,
$$

where the ellipsis corresponds to terms of higher order which stabilise the potential but are negligible during inflation and $V_{\text {top }}$ is a constant density scale. The equation of motion of $\varphi$ is ${ }^{13}$

$$
\ddot{\varphi}+3 H \dot{\varphi}-m_{\varphi}^{2} \varphi \simeq 0,
$$

whose solution has the following growing mode

$$
\varphi \propto a^{-\frac{3}{2}\left[1-\sqrt{1+\frac{4}{9}\left(m_{\varphi} / H\right)^{2}}\right]} .
$$

Therefore, the requirement that $\varphi \propto a$ is achieved if the effective mass of the Higgs field during inflation is

$$
m_{\varphi}=2 H .
$$

It turns out that such a value for the effective mass of the scalar field during inflation is quite reasonable in the context of supergravity theories. Indeed, in Ref. [44] it has been demonstrated that Kähler corrections to the scalar potential are expected to give a contribution of order $H$ to the masses of scalar fields. Hence, these fields would be fast-rolling during inflation down the potential slopes.

Moreover, in this case, $f$ is the gauge kinetic function, which, in supergravity theories, is a holomorphic function of the scalar fields of the theory. Hence, it is natural to expect that $f=f(\varphi)$ and the rolling $\varphi$ would modulate the kinetic function as well as the mass. Indeed, if $m_{\varphi}=2 H$, then to satisfy Eq. (17) one simply needs $f(\varphi) \propto \varphi^{\alpha}$ with $\alpha=-1 \pm 3$.

Of course, $f$ may also depend on other fast-rolling scalar fields $f=f\left(\phi_{1}, \phi_{2}, \ldots \phi_{n}\right)$. If we assume that $f \propto \prod_{i}^{n} \phi_{i}^{\alpha_{i}}$ with $\alpha_{i}=\mathcal{O}(1)$ then

$$
\frac{\dot{f}}{f}=\sum_{i}^{n} \alpha_{i} \frac{\dot{\phi}_{i}}{\phi_{i}}=\frac{3}{2} H \sum_{i}^{n} \alpha_{i}\left(\sqrt{1+\frac{4}{9} \frac{m_{i}^{2}}{H^{2}}}-1\right), \quad \text { where } \quad m_{i}^{2} \equiv \frac{\partial^{2} V}{\partial \phi_{i}^{2}} .
$$

Since Kähler corrections result in $m_{i}^{2} \sim H^{2}$, we find that $\dot{f} / f \sim H$ as required. We would still need to tune $\alpha_{i}$ and $m_{i}^{2}$ such that $\dot{f} / f=(-1 \pm 3) H$, but it is evident that the required values can be naturally attained in the context of supergravity, as also discussed in Ref. [11].

Having said that, since $f$ is the gauge kinetic function in this case, we have $f \sim 1 / e^{2}$, where $e \propto a^{-\alpha / 2}$ is the gauge coupling. This means that, because $f \rightarrow 1$ at the end of inflation, the vector field becomes strongly coupled during inflation if $\alpha>0$. Thus, the physically motivated case is only $\alpha=-4$, where $e \propto a^{2}$ and the vector field is always weakly coupled. Fortunately, it is this case which has the richest phenomenology, as we have shown.

\section{SUMMARY OF RESULTS}

The model studied in this paper, albeit simple, has been shown to have a rich phenomenology, without suffering from any instabilities. By studying particle production in this model we have found that a scale invariant spectrum of vector perturbations for the transverse components can be obtained if $\alpha=-1 \pm 3$ and the field is light at horizon exit. We also found that, to get a scale invariant spectrum of vector field perturbations for the longitudinal component we additionally require that $m \propto a$. We have assumed that this scaling continues throughout inflation as would be the case if both $f$ and $m$ are modulated by some degree of freedom which varies during inflation, e.g. the inflaton field. We also assumed that inflation is quasi-de Sitter.

In all cases we have solved the equations of motion for the mode functions of the components of the vector field perturbations both numerically and through analytic approximations, normalising them appropriately at the vacuum.

13 As in the previous subsection we ignore the backreaction from the vector field. 
A comparison between the two has shown that our analytic expressions approximate the numerical solutions with very high precision. We have found that, after horizon exit, the mode functions $w_{\lambda}$ of the components of the vector field perturbations cease oscillating and follow a power-law evolution of the form

$$
w_{\lambda}=C_{A}+C_{B} a^{-3},
$$

where $C_{A}, C_{B}$ are constants determined by the vacuum initial conditions. Depending on the case considered, the dominant term in the above is either the constant or the decaying mode.

The effective mass of the vector field during inflation is [cf. Eq. (3)]

$$
M=\frac{m}{\sqrt{f}} \propto a^{1-(\alpha / 2)} .
$$

As mentioned, scale invariance for the transverse spectra requires $\alpha=-1 \pm 3$ plus the vector field needs to be light when cosmological scales exit the horizon $M_{*} \ll H_{*}$, where $H_{*} \simeq$ constant is the inflationary Hubble scale. In the case when $\alpha=2$ (i.e. $f \propto a^{2}$ ) we see that $M=$ constant so that $\hat{m}=M_{*} \ll H_{*}$, i.e the vector field remains always light until the end of inflation. In this case, the power-law regime for the evolution of the mode functions of the vector field perturbations is valid until inflation terminates, while the dominant term in Eq. (237) is the constant one for all $w_{\lambda}$ (see Fig. [3).

In contrast, when $\alpha=-4$ (i.e. $f \propto a^{-4}$ ) we have $M \propto a^{3}$. This allows the possibility to have $M_{*} \ll H_{*} \ll \hat{m}$, i.e. while the field is light when the cosmological scales exit the horizon it can become heavy before the end of inflation. If this is the case then the power-law regime is terminated before the end of inflation when the vector field becomes heavy and begins coherent oscillations. Hence, when $\alpha=-4$ we could have either $\hat{m}<H_{*}$ or $\hat{m} \gtrsim H_{*}$. Studying the power-law regime we have shown that the dominant term in Eq. (237) for the mode functions is the constant term for the transverse components but not for the longitudinal one, for which the dominant term is the decaying mode $w_{\|} \propto a^{-3}$ (see Fig. 2). If $\hat{m}<H_{*}$ then inflation ends before the power-law regime is concluded. In this case, the results are the same as in the $\alpha=2$ case. However, if $\hat{m}>H_{*}$ the power-law regime is terminated before the end of inflation.

Consider first that $\hat{m}<H_{*}$, which is possible for $\alpha=-1 \pm 3$. As mentioned, in this case the power-law regime continues until the end of inflation. If this is so we have shown that the power spectra for the transverse and longitudinal components of the vector field superhorizon perturbations are given by

$$
\mathcal{P}_{+}=\left(\frac{H_{*}}{2 \pi}\right)^{2} \quad \text { and } \quad \mathcal{P}_{\|}=\left(\frac{3 H_{*}}{\hat{m}}\right)^{2}\left(\frac{H_{*}}{2 \pi}\right)^{2},
$$

where $\mathcal{P}_{\|}$is the spectrum of the longitudinal component, $\mathcal{P}_{+} \equiv \frac{1}{2}\left(\mathcal{P}_{L}+\mathcal{P}_{R}\right)$, with $\mathcal{P}_{L}, \mathcal{P}_{R}$ being the spectra of the left and right polarisations of the transverse components and $\mathcal{P}_{L}=\mathcal{P}_{R}$ since the model is parity conserving. Because of the condition $\hat{m}<H_{*}$, the particle production process is found to be more efficient in the longitudinal direction than the transverse ones. Hence, in this case, the contribution of the vector field to the curvature perturbation is strongly anisotropic. Indeed, the anisotropy parameter in Eq. (101) in the spectrum is found to be

$$
g=\xi\left(\frac{3 H_{*}}{\hat{m}}\right)^{2}
$$

where $\xi$ is defined in Eq. (106) and quantifies the level of the contribution of the vector field to the overall curvature perturbation $\zeta$ of the Universe. Ref. [12] suggests that $g \lesssim 0.3$, which implies that $\xi \ll 1$ in this case. Hence, the vector field contribution to $\zeta$ has to be subdominant, with the dominant component due to some other source, e.g. the inflaton field.

Non-Gaussianity in the curvature perturbation is found to be also statistically anisotropic with its magnitude and direction correlated with statistical anisotropy in the spectrum as in Ref. 35]. Indeed, for the isotropic part we found [cf. Eq. [124)]

$$
f_{\mathrm{NL}}^{\text {iso }} \simeq \frac{5 g^{2}}{3 \Omega_{A}}\left(\frac{\hat{m}}{3 H_{*}}\right)^{4}=\frac{5 \xi^{2}}{3 \Omega_{\mathrm{dec}}}
$$

which can be substantial even if $\xi \ll 1$, where $\Omega_{\mathrm{dec}}$ is the density parameter at the decay of the vector field. The anisotropic part of $f_{\mathrm{NL}}$ depends on the configuration but we have shown that $\mathcal{G} \gg 1$ in both the equilateral and local cases, where $\mathcal{G}$ is defined in Eq. (120). Thus, we see that the bispectrum is predominantly anisotropic, which suggests that, if non-Gaussianity is observed without significant angular modulation, then the case $\hat{m}<H_{*}$ in our model will be ruled out. 
Consider now that $\hat{m} \gg H_{*}$, which is possible only for $\alpha=-4$. If this is the case, then before the end of inflation the mode functions begin oscillating with frequency increasingly larger than $H_{*}$, while for the average power spectra we have [cf. Eq. (81)]

$$
\overline{\mathcal{P}_{+}}=\overline{\mathcal{P}_{\|}}=\frac{1}{2}\left(\frac{3 H_{*}}{\hat{m}}\right)^{2}\left(\frac{H_{*}}{2 \pi}\right)^{2} .
$$

Since particle production in this case is isotropic there is no statistical anisotropy in the spectrum and the bispectrum, i.e. $g=\mathcal{G}=0$. Therefore, the vector field can alone generate the curvature perturbation of the Universe without any contribution from other sources. The model can indeed generate non-Gaussianity in the curvature perturbation with

$$
f_{\mathrm{NL}}=\frac{5}{4 \hat{\Omega}_{\mathrm{dec}}},
$$

where $\hat{\Omega}_{A}$ is defined in Eq. (110). The above result is identical to the scalar curvaton mechanism. If there are other significant contributions to $\zeta$ beyond the vector field then $f_{\mathrm{NL}}$ is smaller than the above.

It is interesting to consider the case when $\hat{m} \sim H_{*}$, again possible only when $\alpha=-4$ (i.e. $f \propto a^{-4}$ ). In this case the mode functions are about to begin oscillating at the end of inflation, while their value is comparable but not necessarily identical. This case allows the possibility that $|\delta \mathcal{P}|<\mathcal{P}_{+}$, where $\delta \mathcal{P}=\mathcal{P}_{\|}-\mathcal{P}_{+}$. Then, the vector field can alone generate the total curvature perturbation, but it can also produce statistical anisotropy in the spectrum and bispectrum, which would be at equal level

$$
\mathcal{G} \simeq g=\frac{\delta \mathcal{P}}{\mathcal{P}_{+}} \lesssim 0.3 .
$$

In this case we see that the anisotropy in the bispectrum is subdominant, with the dominant component $f_{\mathrm{NL}}^{\text {iso }}$ given by Eq. (243).

To investigate the parameter space for this model we have studied the evolution of the zero-mode of the vector field assuming energy equipartition at the onset of inflation. When $\alpha=2$ we found that $W \simeq$ constant throughout inflation. In contrast, when $\alpha=-4$ we found that the zero mode during inflation scales as $W \propto a^{-3}$ during the power-law regime, while its amplitude scales as $\|W\| \propto a^{-3}$ during the oscillations, when the vector field becomes heavy. In all cases though, the density of the vector field $\rho_{A}$ remains constant during inflation, regardless whether the field is oscillating or not. After inflation, the density scales as radiation $\rho_{A} \propto a^{-4}$ or matter $\rho_{A} \propto a^{-3}$ when the field is light $\hat{m}<H(t)$ or heavy $\hat{m}>H(t)$ respectively. This is different from scalar fields, whose density remains constant when they are light. We have also verified that, when the field is heavy and undergoes oscillations, it acts as a pressureless isotropic fluid, which can dominate the Universe without generating a large-scale anisotropy, in accordance to the findings in Ref. [1].

First, we considered the case when $\hat{m} \gg H_{*}$, possible only if $\alpha=-4$. As there is no anisotropy in this case we assumed that the vector curvaton alone generates $\zeta$. By taking into account all relevant bounds on the decay rate of the inflaton and vector curvaton fields we have found that the scenario works when $H_{*} \gtrsim 10^{9} \mathrm{GeV}$. The vector curvaton begins its oscillations before the end of inflation but no earlier than in the last few e-folds, as $N_{\text {osc }} \lesssim 4$. Still, this allows an exponentially large parameter space for the value of $\hat{m}$, which may be as large as $10^{6} H_{*}$. The parameter space is reduced if the decay of the inflaton is late or if the contribution of the vector field to the energy budget is very small during inflation. This, however, can be counteracted if the vector field decay rate is also small. If $\hat{m} \sim H_{*}$ then the vector field can alone generate $\zeta$ but may also produce statistical anisotropy within the observational bounds. In this case it is easy to show that $H_{*} \gtrsim 10^{9} \mathrm{GeV}$ as well. Finally, the case when $\hat{m}<H_{*}$ allows a slightly lower inflationary scale since the lower bound to $H_{*}$ is relaxed by a factor $<10^{-2} \sqrt{g}$. In this case the vector curvaton contribution to the curvature perturbation is strongly anisotropic, which means that $\Omega_{A} \ll 1$ at decay.

\section{CONCLUSIONS}

In this paper we studied a particularly promising vector curvaton model consisting of a massive Abelian vector field, with a Maxwell type kinetic term and with varying kinetic function $f$ and mass $m$ during inflation. The model is rather generic, it does not suffer from instabilities such as ghosts and may be naturally realised in the context of theories beyond the standard model such as supergravity and superstrings.

We have parametrised the time dependence of the kinetic function as $f \propto a^{\alpha}$, where $a=a(t)$ is the scale factor. Our model offers two distinct possibilities. If $\hat{m}<H_{*}$ (possible for $\alpha=-1 \pm 3$ ) the vector field can only produce 
a subdominant contribution to the curvature perturbation $\zeta$, but it can be the source of statistical anisotropy in the spectrum and bispectrum. In fact, non-Gaussianity in this case is predominantly anisotropic, which means that, if a non-zero $f_{\mathrm{NL}}$ is observed without angular modulation, then our model is falsified in the $\hat{m}<H_{*}$ case. The second possibility (possible for $\alpha=-4$ only) corresponds to $\hat{m} \gtrsim H_{*}$. In this case the vector field can alone generate the curvature perturbation $\zeta$ without any contribution from other sources such as scalar fields. If $\hat{m} \gg H_{*}$ particle production is isotropic and the model does not generate any statistical anisotropy. The vector field begins oscillating a few e-folds before the end of inflation but its density remains constant until inflation ends. The parameter space for this case can be exponentially large, i.e. $1 \ll \hat{m} / H_{*}<10^{6}$. Significant non-Gaussianity can be generated, provided the vector field decays before it dominates the Universe, in which case $f_{\mathrm{NL}}$ is found to be identical to the scalar curvaton scenario. In other words, if $\hat{m} \gg H_{*}$, our vector curvaton can reproduce the results of the scalar curvaton paradigm. Finally, if $\hat{m} \sim H_{*}$ the vector field can alone generate the curvature perturbation $\zeta$ but it can also generate statistical anisotropy in the spectrum and bispectrum. In this case, the anisotropy in $f_{\mathrm{NL}}$ is subdominant and equal to the statistical anisotropy in the spectrum, which is a characteristic signature of this possibility.

We have also found that inflation has to occur at relatively high energies, with $H_{*} \gtrsim 10^{9} \mathrm{GeV}$ in the (almost) isotropic and $H_{*}>\sqrt{g} 10^{7} \mathrm{GeV}$ in the anisotropic case (with $\hat{m} \gtrsim 10 \mathrm{TeV}$ ). These bounds correspond to prompt reheating, with $T_{\mathrm{reh}} \sim V_{*}^{1 / 4}$ which could result in gravitino overproduction. However, if the vector curvaton dominates the Universe, its decay could release enough entropy to efficiently dilute the density of the gravitinos. Furthermore, as in Ref. [11], one could substantially reduce the inflationary scale through introducing an increment to the mass of the vector field, say at a phase transition, after the end of inflation, following the mechanism first suggested for the scalar curvaton scenario in Ref. [45].

For our model to work $f$ and $m$ should vary in a specific manner, which requires tuning. We have outlined two possibilities for achieving the desired modulation for these quantities. First, we considered that the quantities in question are modulated by a string modulus field, which could also play the role of the inflaton. In this case we found that the potential of the modulus has to be approximately exponential, which is reasonable. The second possibility which we discussed was that of a Higgsed vector curvaton in the context of supergravity theories, where scalar fields during inflation obtain an effective mass of order the Hubble scale. We showed that a Higgs field with tachyonic mass $2 H_{*}$ suffices to account for the desired modulation for $m$, while it is natural to expect that the gauge kinetic function is modulated by the fast-rolling scalar fields of the theory such that $\dot{f} / f \sim H_{*}$ as required. These examples demonstrate that the tuning of the modulation of $f$ and $m$ can be attained in a realistic manner. This should be contrasted with the traditional case of generating the curvature perturbation using scalar fields, where their effective mass needs to be fine-tuned at least by $\mathcal{O}\left(10^{-2}\right)$ against Kähler corrections to produce an approximately scale-invariant spectrum (the famous $\eta$-problem). In our model, the vector field also needs to be effectively massless $\left(M_{*}<H_{*}\right)$ when the cosmological scales exit the horizon but, to our knowledge, there is no compelling reason why this should not be so. Note also, that the vector field can become heavy by the end of inflation in the $\alpha=-4$ case. We considered de Sitter inflation in our treatment so that the obtained spectra were exactly scale invariant if $f \propto a^{-1 \pm 3}$ and $m \propto a$. Deviations from the Harrison-Zel'dovich spectrum can be attained either by perturbing the modulation of $f$ and $m$ or by considering quasi-de Sitter inflation, with $\varepsilon \equiv-\dot{H} / H^{2} \sim 10^{-2}$.

Even though in our specific examples we employed scalar fields to modulate $f$ and $m$, in principle their variation can be controlled by any kind of degree of freedom which varies during inflation. In that sense, this model can generate the curvature perturbation in the Universe without direct involvement of fundamental scalar fields. Of course, theories beyond the standard model are abundant with scalar fields and vector fields alike. The next step, therefore, is to realise this vector curvaton model in the context of realistic extensions of the standard model.

\section{Acknowledgments}

This work was supported (in part) by the European Union through the Marie Curie Research and Training Network "UniverseNet" (MRTN-CT-2006-035863) and by STFC Grant ST/G000549/1. M.K. and J.M.W. are also supported by the Lancaster University Physics Department.

\section{References}

[1] K. Dimopoulos, Phys. Rev. D 74 (2006) 083502.

[2] D. H. Lyth and D. Wands, Phys. Lett. B 524 (2002) 5; K. Enqvist and M. S. Sloth, Nucl. Phys. B 626 (2002) 395; T. Moroi and T. Takahashi, Phys. Lett. B 522 (2001) 215 [Erratum-ibid. B 539 (2002) 303].

[3] S. Mollerach, Phys. Rev. D 42, 313 (1990); A. D. Linde and V. Mukhanov, Phys. Rev. D 56, R535 (1997). 
[4] K. Dimopoulos and D. H. Lyth, Phys. Rev. D 69 (2004) 123509; T. Moroi, T. Takahashi and Y. Toyoda, Phys. Rev. D 72 (2005) 023502; T. Moroi and T. Takahashi, Phys. Rev. D 72 (2005) 023505.

[5] D. Grasso and H.R. Rubinstein, Phys. Rept. 348 (2001) 163; M. Giovannini, Int. J. Mod. Phys. D 13 (2004) 391.

[6] M. S. Turner and L. M. Widrow, Phys. Rev. D 37 (1988) 2743.

[7] A. C. Davis, K. Dimopoulos, T. Prokopec and O. Tornkvist, Phys. Lett. B 501 (2001) 165; K. Dimopoulos, T. Prokopec, O. Tornkvist and A. C. Davis, Phys. Rev. D 65 (2002) 063505.

[8] W. D. Garretson, G. B. Field and S. M. Carroll, Phys. Rev. D 46 (1992) 5346; F. D. Mazzitelli and F. M. Spedalieri, Phys. Rev. D 52 (1995) 6694; M. Novello, L. A. R. Oliveira and J. M. Salim, Class. Quant. Grav. 13 (1996) 1089; A. Dolgov, Phys. Rev. D 48 (1993) 2499; B. Ratra, Astrophys. J. 391 (1992) L1; E. A. Calzetta, A. Kandus and F. D. Mazzitelli, Phys. Rev. D 57 (1998) 7139; O. Bertolami and D. F. Mota, Phys. Lett. B 455 (1999) 96; M. Giovannini, Phys. Rev. D 62 (2000) 123505; T. Prokopec and E. Puchwein, JCAP 0404 (2004) 007; Phys. Rev. D 70 (2004) 043004; K. Enqvist, A. Jokinen and A. Mazumdar, JCAP 0411 (2004) 001; M. R. Garousi, M. Sami and S. Tsujikawa, Phys. Lett. B 606 (2005) 1; A. Ashoorioon and R. B. Mann, Phys. Rev. D 71 (2005) 103509; J. E. Madriz Aguilar and M. Bellini, Phys. Lett. B 642 (2006) 302; F. Agustin Membiela and M. Bellini, 0712.3032 [hep-th]; L. Campanelli, P. Cea, G. L. Fogli and L. Tedesco, Phys. Rev. D 77 (2008) 043001; Phys. Rev. D 77 (2008) 123002.

[9] M. Giovannini, Phys. Rev. D 64 (2001) 061301; K. Bamba and J. Yokoyama, Phys. Rev. D 69 (2004) 043507; Phys. Rev. D 70 (2004) 083508; O. Bertolami and R. Monteiro, Phys. Rev. D 71 (2005) 123525; J. M. Salim, N. Souza, S. E. Perez Bergliaffa and T. Prokopec, JCAP 0704 (2007) 011; K. Bamba and M. Sasaki, JCAP 0702 (2007) 030; J. Martin and J. Yokoyama, JCAP 0801 (2008) 025; K. Bamba and S. D. Odintsov, JCAP 0804 (2008) 024; K. Bamba, C. Q. Geng and S. H. Ho, JCAP 0811 (2008) 013; V. Demozzi, V. Mukhanov and H. Rubinstein, JCAP 0908 (2009) 025.

[10] K. Dimopoulos and M. Karčiauskas, JHEP 0807 (2008) 119.

[11] K. Dimopoulos, Phys. Rev. D 76 (2007) 063506.

[12] N. E. Groeneboom and H. K. Eriksen, Astrophys. J. 690 (2009) 1807; N. E. Groeneboom, L. Ackerman, I. K. Wehus and H. K. Eriksen, arXiv:0911.0150 [astro-ph.CO].

[13] A. R. Pullen and M. Kamionkowski, Phys. Rev. D 76 (2007) 103529.

[14] K. Land and J. Magueijo, Phys. Rev. Lett. 95 (2005) 071301.

[15] A. Slosar et al., 0809.0717 [astro-ph].

[16] M. J. Longo, astro-ph/0703325.

[17] A. de Oliveira-Costa and M. Tegmark, Phys. Rev. D 74 (2006) 023005.

[18] H. K. Eriksen, F. K. Hansen, A. J. Banday, K. M. Gorski and P. B. Lilje, Astrophys. J. 605 (2004) 14 [Erratumibid. 609 (2004) 1198]; F. K. Hansen, A. J. Banday and K. M. Gorski, Mon. Not. Roy. Astron. Soc. 354 (2004) 641.

[19] S. Yokoyama and J. Soda, JCAP 0808 (2008) 005.

[20] L. Ackerman, S. M. Carroll and M. B. Wise, Phys. Rev. D 75 (2007) 083502.

[21] S. Kanno, M. Kimura, J. Soda and S. Yokoyama, JCAP 0808 (2008) 034; M. A. Watanabe, S. Kanno and J. Soda, Phys. Rev. Lett. 102 (2009) 191302.

[22] K. Dimopoulos, M. Karčiauskas, D. H. Lyth and Y. Rodriguez, JCAP 0905 (2009) 013.

[23] A. A. Starobinsky, JETP Lett. 42 (1985) 152 [Pisma Zh. Eksp. Teor. Fiz. 42 (1985) 124]; M. Sasaki and E. D. Stewart, Prog. Theor. Phys. 95 (1996) 71; D. H. Lyth, K. A. Malik and M. Sasaki, JCAP 0505 (2005) 004.

[24] M. C. Bento, O. Bertolami, P. V. Moniz, J. M. Mourao and P. M. Sa, Class. Quant. Grav. 10 (1993) 285.

[25] L. H. Ford, Phys. Rev. D 40 (1989) 967; C. M. Lewis, Phys. Rev. D 44 (1991) 1661. 
[26] A. Golovnev, V. Mukhanov and V. Vanchurin, JCAP 0806 (2008) 009; JCAP 0811 (2008) 018; T. Chiba, JCAP 0808 (2008) 004; A. Golovnev and V. Vanchurin, Phys. Rev. D 79 (2009) 103524; A. Golovnev, 0910.0173 [astro-ph.CO].

[27] Y. Zhang, Phys. Rev. D 80 (2009) 043519.

[28] T. S. Koivisto and D. F. Mota, JCAP 0808 (2008) 021.

[29] A. Tartaglia and N. Radicella, Phys. Rev. D 76 (2007) 083501. S. Koh and B. Hu, 0901.0429 [hep-th]; S. Koh, 0902.3904 [hep-th].

[30] C. Armendariz-Picon, JCAP 0407 (2004) 007; H. Wei and R. G. Cai, Phys. Rev. D 73 (2006) 083002; C. G. Boehmer and T. Harko, Eur. Phys. J. C 50 (2007) 423; T. Koivisto and D. F. Mota, Astrophys. J. 679 (2008) 1; H. Wei and R. G. Cai, JCAP 0709 (2007) 015; J. B. Jimenez and A. L. Maroto, Phys. Rev. D 78 (2008) 063005; 0807.2528 [astro-ph]; AIP Conf. Proc. 1122 (2009) 107; Phys. Rev. D 80 (2009) 063512; J. B. Jimenez, R. Lazkoz and A. L. Maroto, 0904.0433 [astro-ph.CO].

[31] K. Bamba, S. Nojiri and S. D. Odintsov, Phys. Rev. D 77 (2008) 123532; K. Bamba and S. Nojiri, 0811.0150 [hep-th].

[32] W. Zhao and Y. Zhang, Class. Quant. Grav. 23 (2006) 3405; W. Zhao, Int. J. Mod. Phys. D 16 (2007) 1735; 0810.5506 [gr-qc].

[33] C. Germani and A. Kehagias, JCAP 0903 (2009) 028; JCAP 0911 (2009) 005; T. Kobayashi and S. Yokoyama, JCAP 0905 (2009) 004; T. S. Koivisto, D. F. Mota and C. Pitrou, JHEP 0909 (2009) 092; T. S. Koivisto and N. J. Nunes, Phys. Rev. D 80 (2009) 103509; 0907.3883 [astro-ph.CO].

[34] C. A. Valenzuela-Toledo and Y. Rodriguez, 0910.4208 [astro-ph.CO].

[35] M. Karčiauskas, K. Dimopoulos and D. H. Lyth, Phys. Rev. D 80 (2009) 023509.

[36] N. Bartolo, E. Dimastrogiovanni, S. Matarrese and A. Riotto, JCAP 0910 (2009) 015; 0909.5621 [astro-ph.CO].

[37] B. Himmetoglu, C. R. Contaldi and M. Peloso, Phys. Rev. D 79 (2009) 063517; Phys. Rev. Lett. 102 (2009) 111301. 0909.3524 [astro-ph.CO].

[38] S. M. Carroll, T. R. Dulaney, M. I. Gresham and H. Tam, Phys. Rev. D 79 (2009) 065011; T. R. Dulaney, M. I. Gresham and M. B. Wise, Phys. Rev. D 77 (2008) 083510 [Erratum-ibid. D 79 (2009) 029903];

[39] K. Dimopoulos, M. Karciauskas and J. M. Wagstaff, 0909.0475 [hep-ph].

[40] J. M. Cornwall, D. N. Levin and G. Tiktopoulos, Phys. Rev. D 10 (1974) 1145 [Erratum-ibid. D 11 (1975) 972].

[41] E. Komatsu et al. [WMAP Collaboration], Astrophys. J. Suppl. 180 (2009) 330.

[42] C. A. Valenzuela-Toledo, Y. Rodriguez and D. H. Lyth, 0909.4064 [astro-ph.CO].

[43] I. Sollom, A. Challinor and M. P. Hobson, Phys. Rev. D 79 (2009) 123521.

[44] M. Dine, L. Randall and S. Thomas, Nucl. Phys. B 458 (1996) 291; Phys. Rev. Lett. 75 (1995) 398; D. H. Lyth and T. Moroi, JHEP 0405 (2004) 004.

[45] K. Dimopoulos, D. H. Lyth and Y. Rodriguez, JHEP 0502 (2005) 055. 